\begin{tabular}{|c|c|}
\hline Title & $\begin{array}{l}\text { Formulation of the spherical harmonic coefficients of the entire magnetic field components generated by magnetic } \\
\text { moment and current for shimming }\end{array}$ \\
\hline Author(s) & Noguchi, So \\
\hline Citation & $\begin{array}{l}\text { Journal of A pplied Physics, 115(16), } 163908 \\
\text { https://doi. org/10.1063/1.4872244 }\end{array}$ \\
\hline Issue Date & $2014-0428$ \\
\hline Doc URL & http:/hdl. handle.net/2115/56746 \\
\hline Rights & $\begin{array}{l}\text { Copyright } 2014 \text { A merican Institute of Phy sics. This article may be downloaded for personal use only. A ny other use } \\
\text { requires prior permission of the author and the A merican Institute of Physics. The following article appeared in J. A ppl. } \\
\text { Phys. 115, 163908(2014) and may be found at http://scitation.aip.org/content/aip } \$ \text { ournal } / \text { ap } / 115 / 16 / 10.1063 / 1.48722244\end{array}$ \\
\hline Type & article \\
\hline File Information & 1.4872244.pdf \\
\hline
\end{tabular}

Instructions for use 


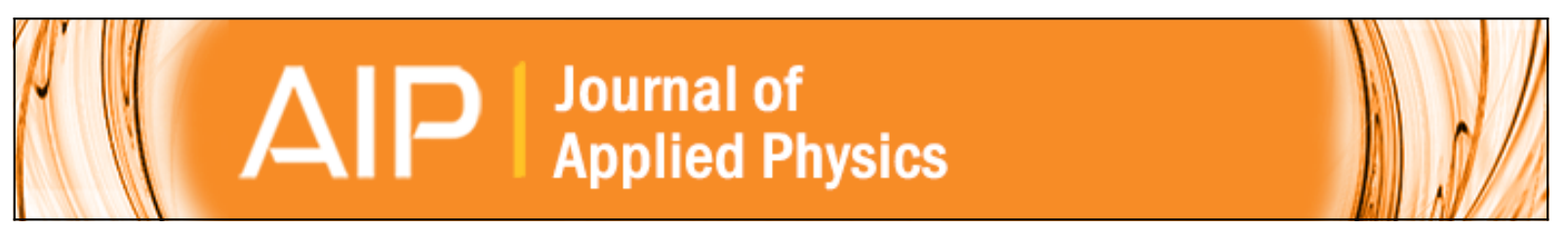

\section{Formulation of the spherical harmonic coefficients of the entire magnetic field components generated by magnetic moment and current for shimming}

So Noguchi

Citation: Journal of Applied Physics 115, 163908 (2014); doi: 10.1063/1.4872244

View online: http://dx.doi.org/10.1063/1.4872244

View Table of Contents: http://scitation.aip.org/content/aip/journal/jap/115/16?ver=pdfcov

Published by the AIP Publishing

\section{Articles you may be interested in}

The random dipolar-field approximation for systems of interacting magnetic particles

J. Appl. Phys. 113, 163908 (2013); 10.1063/1.4802583

Magnetic moment manipulation by triplet Josephson current

Appl. Phys. Lett. 101, 242602 (2012); 10.1063/1.4769900

Structural and magnetic characterization of superparamagnetic iron platinum nanoparticle contrast agents for magnetic resonance imaging

J. Vac. Sci. Technol. B 30, 02C101 (2012); 10.1116/1.3692250

Effect of localized magnetic field on the uniform ferromagnetic resonance mode in a thin film Appl. Phys. Lett. 94, 172508 (2009); 10.1063/1.3123264

Calibration of magnetic force microscopy tips by using nanoscale current-carrying parallel wires J. Appl. Phys. 95, 775 (2004); 10.1063/1.1633979

\section{Advances in Live Single-Cell Thermal Imaging and Manipulation} International Symposium, November 10-12, 2014

biophysics; soft condensed matter/soft mesoscopics; IR/terahertz spectroscopy

single-molecule optoelectronics/nanoplasmonics; photonics; living matter physics

\section{Application deadline: August 24}

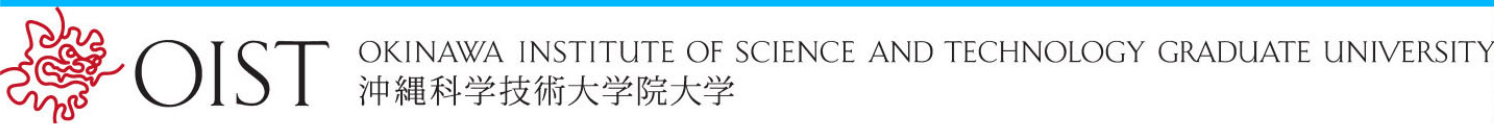

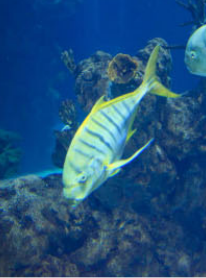




\title{
Formulation of the spherical harmonic coefficients of the entire magnetic field components generated by magnetic moment and current for shimming
}

\author{
So Noguchi ${ }^{\text {a) }}$ \\ Graduate School of Information Science and Technology, Hokkaido University, Kita 14 Nishi 9 , \\ Kita-ku, Sapporo 060-0814, Japan
}

(Received 5 February 2014; accepted 11 April 2014; published online 28 April 2014)

\begin{abstract}
For MRI and NMR magnet design, a highly homogeneous and high magnetic field has to be achieved by active and/or passive shimming. The active and passive shimming commonly homogenize the magnetic field around the magnet center by cancellation of the higher terms of a spherical harmonic series than the 0th term. So far, the spherical harmonic expression in the cylindrical coordinate system is well known for a circular coil, a solenoid coil, and magnetization of ferromagnetic material with/without its volume. In a shimming design, only the $z$-component of an inhomogeneous magnetic field is compensated because it is dominant to the performance of MRI/NMR. However, various kinds of MRI and NMR systems have recently been developed, and these magnets sometimes have an axially asymmetric configuration or generate a tilted magnetic field. Therefore, the entire $x$-, $y$-, and $z$-components have to be homogenized for such magnets. I derive the spherical harmonic expression of the entire components of magnetic field generated by a circular coil, a dipole coil, and a straight line current. In addition, since the entire magnetization components of the ferromagnetic material having the volume contribute the entire components of the magnetic field around the magnet center, I also derive the equations of the spherical harmonic coefficients of the magnetic field generated by ferromagnetic material. Since these equations need a numerical integration, such as the Gauss quadrature integration, the computation accuracy of the spherical harmonic coefficients is investigated against the number of the evaluation points. We can calculate the highly accurate spherical harmonic coefficients with the small number of the evaluation points. (C) 2014 AIP Publishing LLC. [http://dx.doi.org/10.1063/1.4872244]
\end{abstract}

\section{INTRODUCTION}

Commercial MRI and NMR systems having highly homogeneous magnetic field have been developed since 1970s. The magnetic field of MRI and NMR has strengthened, and they give a high image resolution. In recent years, MRI and NMR systems are widely used, and new kinds of MRI/NMR, such as functional MRI and magic-angle-spinning NMR, have been innovated. The MRI/NMR systems are advanced with the development of the superconducting magnet technology. ${ }^{1}$ Currently, the high field MRI using high-Tc superconducting magnet of approximately $10 \mathrm{~T}$ is under development by some institutes. ${ }^{2,3}$ In addition, a magnetic field correction and a shimming technique are also requisite for a highly homogeneous magnetic field.

A shimming technique has been developed with the development of MRI/NMR systems. When shimming a magnet field, the higher terms of the spherical harmonic series than the 0th term, generated by the main magnet, are compensated by shim coils. It is called "active shimming." The simplest active shimming technique is the use of one-turn circular coil, whose volume is ignorable. The spherical harmonic coefficients of one-turn circular coil, such as z2 and z4 shim coils, were given by many papers. ${ }^{4,5}$ Some papers ${ }^{6-8}$ gave the expression of the spherical harmonic coefficients to the cylindrical coil with volume, such as a solenoid coil.
Although the active shimming with coils is relatively convenient and easy, a shimming with ferromagnetic material, called "passive shimming," is highly flexible and effective. The magnetic moment of the ferromagnetic material, like a magnetic dipole without volume, compensates the magnetic field of the main magnet. ${ }^{4,9-11}$ Lopez et al. gave the spherical harmonic expression of the magnetized ferromagnetic material with volume. ${ }^{12}$ However, it did not show a method of calculating the magnetization. On the other hand, by employing the equivalent magnetization current method, the spherical harmonic expression was also derived to the magnetized ferromagnetic material considering its volume, with computation method of the magnetization taking into account its non-linear properties. ${ }^{13}$

Subsequently, methods of configuring small pieces of ferromagnetic material were proposed. ${ }^{14,15}$ They employed the expressing of spherical harmonics and a magnetization computation as well as an optimization method. I proposed a method of passive shimming in addition to magnetic shielding. ${ }^{16,17}$ The above calculation methods and optimization ones facilitate a first-cut design of the passive shimming.

So far, the active and passive shimming to the axially symmetric magnet were discussed on the papers. ${ }^{4-17}$ The $z$-component of the magnetic field, parallel to the axis of the symmetry, is homogenized, and the others (radial and azimuthal components) are ignored. Meanwhile, various kinds of MRI magnet have recently been developed, such as an open type $\mathrm{MRI}^{18}$ and a magic-angle-spinning NMR. ${ }^{19}$ These systems generate an axially asymmetric magnetic field because of 
axially asymmetric configuration of a magnet or a return yoke, without shimming. Kakugawa et al. derived the spherical harmonic expression for the entire components of a magnetic moment. ${ }^{18}$ The expression took into account the volume of ferromagnetic material piece, whereas only the $z$-component of the expression was given. The magnetic field of the magicangle-spinning NMR, however, tilts from the $z$-axis. ${ }^{19}$ For passive shimming of the magic-angle-spinning NMR, the spherical harmonic expression of the entire components of the magnetic field in the Cartesian coordinate system is required to all the magnetic moment components of a magnetized ferromagnetic material piece having the volume.

Consequently, I have derived the spherical harmonic expression of the entire components of the magnetic field for all the magnetic moment components of a magnetized ferromagnetic material piece with the volume. In addition, the spherical harmonic expression of the entire components of various coils, such as a circular coil, a solenoid coil, a straight current, and a dipole coil, is also shown in this paper. Since the developed equations involve integration forms, it is necessary to calculate them with a numerical integration method. Hence, the calculation accuracy to the numerical integration is investigated against the number of the evaluation points. It is confirmed that the high accuracy is achieved with a relatively small number of evaluation points, except a straight line current. The derived spherical harmonic expression is effective in the design of active and/or passive shimming.

\section{FORMULATION OF SPHERICAL HARMONIC EXPRESSION OF MAGNETIC FIELD}

In order to evaluate the homogeneity of a magnetic field $\boldsymbol{B}(\boldsymbol{r})$, it is necessary to take into account a magnetic field $\boldsymbol{B}_{\mathrm{c}}(\boldsymbol{r})$ produced by currents and a magnetic field $\boldsymbol{B}_{\mathrm{m}}(\boldsymbol{r})$ generated by pieces of ferromagnetic material.

$$
\boldsymbol{B}(\boldsymbol{r})=\boldsymbol{B}_{\mathrm{c}}(\boldsymbol{r})+\boldsymbol{B}_{\mathrm{m}}(\boldsymbol{r}) .
$$

The magnetic field $\boldsymbol{B}(\boldsymbol{r})$ around the center of a magnet system can be expressed by a spherical harmonic representation. For homogenizing the magnetic field, the higher-order coefficients of the spherical harmonics of the magnetic field generated by the current and the ferromagnetic materials than 0th order have to be eliminated. Therefore, it is necessary to represent the magnetic fields $\boldsymbol{B}_{\mathrm{c}}(\boldsymbol{r})$ and $\boldsymbol{B}_{\mathrm{m}}(\boldsymbol{r})$ as the spherical harmonic function.

The formulation of the spherical harmonic expression of magnetic field generated by magnetic moments and currents is described in the following sections.

\section{A. Magnetic field generated by dipole magnetic moment}

A dipole magnetic moment $\boldsymbol{m}$ at a point $\mathrm{Q}$ generates a magnetic flux $\Phi$ at a point P (Fig. 1). The magnetic flux $\Phi$ is expressed by

$$
\Phi=-\frac{d \boldsymbol{m}}{4 \pi \mu_{0}} \cdot \nabla_{\mathrm{Q}}\left(\frac{1}{R}\right)
$$

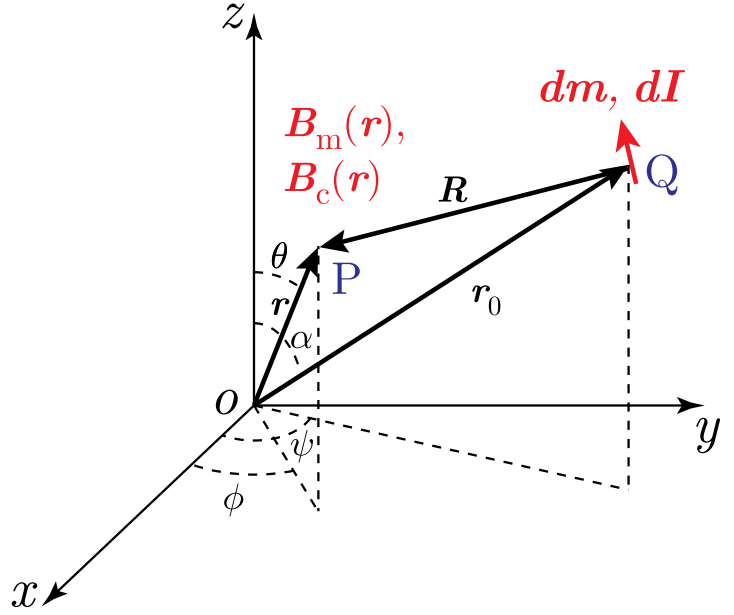

FIG. 1. Magnetic field $\boldsymbol{B}_{\mathrm{m}}(\boldsymbol{r})$ or $\boldsymbol{B}_{\mathrm{c}}(\boldsymbol{r})$ at point $\mathrm{P}$ is generated by dipole magnetic moment $\boldsymbol{d} \boldsymbol{m}$ or dipole current $\boldsymbol{d} \boldsymbol{I}$ at point $\mathrm{Q}$, respectively.

where $\mu_{0}$ and $R$ are the permeability of free space and the distance between the points $\mathrm{P}$ and $\mathrm{Q}$, respectively. The function $1 / R$ in spherical harmonics is given using a Green function $^{4}$ by

$$
\begin{aligned}
\frac{1}{R}= & \sum_{n=0}^{\infty} \sum_{m=0}^{n} \varepsilon_{m} \frac{(n-m) !}{(n+m) !} P_{n}^{m}(\cos \alpha) P_{n}^{m}(\cos \theta) \\
& \times \cos [m(\phi-\psi)] \begin{cases}r_{0}^{n} / r^{n+1} & r>r_{0} \\
r^{n} / r_{0}^{n+1} & r<r_{0},\end{cases}
\end{aligned}
$$

where $\varepsilon_{m}, P_{n}^{m}, r$, and $r_{0}$ are the Nuemann factor $\left(\varepsilon_{m}=1\right.$ if $m=0$, otherwise $\varepsilon_{m}=2$ ), the associated Legendre function, and the position vectors of $\mathrm{P}$ and $\mathrm{Q}$, respectively. The angles $\alpha, \theta, \phi$, and $\psi$ are shown in Fig. 1. The associated Legendre functions are shown in Appendix A.

Let

$$
F_{n m}=\varepsilon_{m} \frac{(n-m) !}{(n+m) !}
$$

and

$$
T_{n m}^{\prime}=P_{n}^{m}(\cos \alpha) \cos [m(\phi-\psi)] \begin{cases}r_{0}^{n} & r>r_{0} \\ 1 / r_{0}^{n+1} & r<r_{0}\end{cases}
$$

Eq. (2) becomes

$$
\Phi=-\frac{\boldsymbol{d} \boldsymbol{m}}{4 \pi \mu_{0}} \cdot F_{n m} P_{n}^{m}(\cos \theta) \nabla_{\mathrm{Q}} T_{n m}^{\prime}\left\{\begin{array}{ll}
1 / r^{n+1} & r>r_{0} \\
r^{n} & r<r_{0}
\end{array} .\right.
$$

Here,

$$
\nabla_{\mathrm{Q}} T_{n m}^{\prime}=\frac{\partial T_{n m}^{\prime}}{\partial x} \boldsymbol{i}+\frac{\partial T_{n m}^{\prime}}{\partial y} \boldsymbol{j}+\frac{\partial T_{n m}^{\prime}}{\partial z} \boldsymbol{k}
$$

where $\boldsymbol{i}, \boldsymbol{j}$, and $\boldsymbol{k}$ are the unit vector in the $x$-, $y$-, and $z$-directions. The partial differential of the $T_{n m}^{\prime}$ is given as 


$$
\begin{gathered}
\frac{\partial T_{n m}^{\prime}}{\partial x}=\frac{r_{0}^{n-1}}{2}\left\{(n+m)(n+m-1) \times P_{n-1}^{m-1}(\cos \alpha) \cos [(m-1) \psi-m \phi]-P_{n-1}^{m+1}(\cos \alpha) \cos [(m+1) \psi-m \phi]\right\} \\
\frac{\partial T_{n m}^{\prime}}{\partial y}=\frac{r_{0}^{n-1}}{2}\left\{-(n+m)(n+m-1) \times P_{n-1}^{m-1}(\cos \alpha) \sin [(m-1) \psi-m \phi]-P_{n-1}^{m+1}(\cos \alpha) \sin [(m+1) \psi-m \phi]\right\} \\
\frac{\partial T_{n m}^{\prime}}{\partial z}=r_{0}^{n-1}(n+m) P_{n-1}^{m}(\cos \alpha) \times \cos [m(\psi-\phi)]
\end{gathered}
$$

when $r>r_{0}$, and

$$
\begin{gathered}
\frac{\partial T_{n m}^{\prime}}{\partial x}=-\frac{1}{2 r_{0}^{n+2}}\left\{-(n-m+1)(n-m+2) \times P_{n+1}^{m-1}(\cos \alpha) \cos [(m-1) \psi-m \phi]+P_{n+1}^{m+1}(\cos \alpha) \cos [(m+1) \psi-m \phi]\right\} \\
\frac{\partial T_{n m}^{\prime}}{\partial y}=-\frac{1}{2 r_{0}^{n+2}}\left\{(n-m+1)(n-m+2) \times P_{n+1}^{m-1}(\cos \alpha) \sin [(m-1) \psi-m \phi]+P_{n+1}^{m+1}(\cos \alpha) \sin [(m+1) \psi-m \phi]\right\} \\
\frac{\partial T_{n m}^{\prime}}{\partial z}=-\frac{1}{r_{0}^{n+2}}(n-m+1) P_{n+1}^{m}(\cos \alpha) \times \cos [m(\psi-\phi)]
\end{gathered}
$$

when $r<r_{0}$. The derivation is shown in Appendix B.

The magnetic field $\boldsymbol{d} \boldsymbol{B}_{\mathrm{m}}(\boldsymbol{r})$ generated by the dipole magnetic moment $\boldsymbol{d} \boldsymbol{m}$ is

$$
\boldsymbol{d} \boldsymbol{B}_{\mathrm{m}}(\boldsymbol{r})=-\mu_{0} \nabla_{\mathrm{P}} \Phi
$$

Let $\boldsymbol{d} \boldsymbol{m}=\left(d m_{\rho}, d m_{\phi}, d m_{z}\right)$ with the cylindrical components, so that we can obtain the entire components of the magnetic field for each component of the dipole magnetic moment. Here, when $r<r_{0}$, let

$$
\begin{aligned}
Y_{n m}^{\prime} & =P_{n}^{m}(\cos \theta) \cos [m(\psi-\phi)] r^{n} \\
Y_{n m}^{\prime \prime} & =P_{n}^{m}(\cos \theta) \sin [m(\psi-\phi)] r^{n} .
\end{aligned}
$$

The parietal differential of $Y_{n m}^{\prime}$ is obtained like Eqs. (8)-(10), and that of $Y_{n m}^{\prime \prime}$ is as follows

$$
\begin{gathered}
\frac{\partial Y_{n m}^{\prime \prime}}{\partial x}=-\frac{r^{n-1}}{2}\left\{(n+m)(n+m-1) P_{n-1}^{m-1}(\cos \theta) \times \sin [(m-1) \phi-m \psi]-P_{n-1}^{m+1}(\cos \theta) \times \sin [(m+1) \phi-m \psi]\right\} \\
\frac{\partial Y_{n m}^{\prime \prime}}{\partial y}=-\frac{r^{n-1}}{2}\left\{(n+m)(n+m-1) P_{n-1}^{m-1}(\cos \theta) \times \cos [(m-1) \phi-m \psi]+P_{n-1}^{m+1}(\cos \theta) \times \cos [(m+1) \phi-m \psi]\right\} \\
\frac{\partial Y_{n m}^{\prime \prime}}{\partial z}=r^{n-1}(n+m) P_{n-1}^{m}(\cos \theta) \sin [m(\psi-\phi)] .
\end{gathered}
$$

When the components of the magnetic field $\boldsymbol{d} \boldsymbol{B}_{\mathrm{m}}(\boldsymbol{r})$ are $\left(d B_{\mathrm{m} x}, d B_{\mathrm{m} y}, d B_{\mathrm{m} z}\right)$ in the Cartesian coordinate system, with Eqs. (8)-(10) and (17)-(19), the entire components of the magnetic field generated by the dipole magnetic moment are given as follows $\left(r<r_{0}\right)$ :

$$
\begin{aligned}
& d B_{\mathrm{m} x}=d B_{\mathrm{m} x, \rho}+d B_{\mathrm{m} x, \phi}+d B_{\mathrm{m} x, z}, \\
& d B_{\mathrm{m} y}=d B_{\mathrm{m} y, \rho}+d B_{\mathrm{m} y, \phi}+d B_{\mathrm{m} y, z}, \\
& d B_{\mathrm{m} z}=d B_{\mathrm{m} z, \rho}+d B_{\mathrm{m} z, \phi}+d B_{\mathrm{m} z, z},
\end{aligned}
$$


where

$$
\begin{aligned}
& d B_{\mathrm{m} x, \rho}=\frac{d m_{\rho}}{4 \pi}\left[\frac{1}{2 r_{0}^{3}}\left(P_{2}^{2}(\cos \alpha)-2 P_{2}(\cos \alpha)\right) \cos \psi-\sum_{n=2}^{\infty} \frac{r^{n-1}}{r_{0}^{n+2}} P_{n+1}^{1}(\cos \alpha) P_{n-1}^{1}(\cos \theta) \cos \phi+\sum_{n=2}^{\infty} \sum_{m=1}^{n} \frac{1}{2} \frac{(n-m) !}{(n+m) !} \frac{r^{n-1}}{r_{0}^{n+2}}\right. \\
& \times\left\{P_{n+1}^{m+1}(\cos \alpha)-(n-m+1)(n-m+2) P_{n+1}^{m-1}(\cos \alpha)\right\}\left\{(n+m)(n+m-1) P_{n-1}^{m-1}(\cos \theta)\right. \\
& \left.\times(\cos m \psi \cos (m-1) \phi+\sin m \psi \sin (m-1) \phi)-P_{n-1}^{m+1}(\cos \theta)(\cos m \psi \cos (m+1) \phi+\sin m \psi \sin (m+1) \phi\}\right], \\
& d B_{\mathrm{m} x, \phi}=\frac{d m_{\phi}}{4 \pi}\left[\frac{1}{r_{0}^{3}} \sin \psi-\sum_{n=2}^{\infty} \sum_{m=1}^{n} \frac{1}{2} \frac{(n-m) !}{(n+m) !} r_{0}^{n-1} r_{n+1}^{n+2}\left\{P_{n+1}^{m+1}(\cos \alpha)+(n-m+1)(n-m+2) P_{n+1}^{m-1}(\cos \alpha)\right\}\right. \\
& \times\left\{(n+m)(n+m-1) P_{n-1}^{m-1}(\cos \theta)(\cos m \psi \sin (m-1) \phi-\sin m \psi \cos (m-1) \phi)\right. \\
& \left.-P_{n-1}^{m+1}(\cos \theta)(\cos m \psi \sin (m+1) \phi-\sin m \psi \cos (m+1) \phi\}\right] \text {, } \\
& d B_{\mathrm{m} x, z}=\frac{d m_{z}}{4 \pi}\left[-\sum_{n=2}^{\infty}(n+1) \frac{r^{n-1}}{r_{0}^{n+2}} P_{n+1}(\cos \alpha) P_{n-1}^{1}(\cos \theta) \cos \phi+\sum_{n=1}^{\infty} \sum_{m=1}^{n} \frac{(n-m) !}{(n+m) !} \frac{r^{n-1}}{r_{0}^{n+2}}(n-m+1) P_{n+1}^{m}(\cos \alpha)\right. \\
& \times\left\{(n+m)(n+m-1) P_{n-1}^{m-1}(\cos \theta)(\cos m \psi \cos (m-1) \phi+\sin m \psi \sin (m-1) \phi)\right. \\
& \left.-P_{n-1}^{m+1}(\cos \theta)(\cos m \psi \cos (m+1) \phi+\sin m \psi \sin (m+1) \phi\}\right], \\
& d B_{\mathrm{m} y, \rho}=\frac{d m_{\rho}}{4 \pi}\left[\frac{1}{2 r_{0}^{3}}\left(P_{2}^{2}(\cos \alpha)-2 P_{2}(\cos \alpha)\right) \sin \psi-\sum_{n=2}^{\infty} \frac{r^{n-1}}{r_{0}^{n+2}} P_{n+1}^{1}(\cos \alpha) P_{n-1}^{1}(\cos \theta) \sin \phi\right. \\
& -\sum_{n=2}^{\infty} \sum_{m=1}^{n} \frac{1}{2} \frac{(n-m) !}{(n+m) !} \frac{r^{n-1}}{r_{0}^{n+2}}\left\{P_{n+1}^{m+1}(\cos \alpha)-(n-m+1)(n-m+2) P_{n+1}^{m-1}(\cos \alpha)\right\} \\
& \times\left\{(n+m)(n+m-1) P_{n-1}^{m-1}(\cos \theta)(\cos m \psi \sin (m-1) \phi-\sin m \psi \cos (m-1) \phi)\right. \\
& \left.+P_{n-1}^{m+1}(\cos \theta)(\cos m \psi \sin (m+1) \phi-\sin m \psi \cos (m+1) \phi\}\right], \\
& d B_{\mathrm{m} y, \phi}=\frac{d m_{\phi}}{4 \pi}\left[-\frac{1}{r_{0}^{3}} \cos \psi-\sum_{n=2}^{\infty} \sum_{m=1}^{n} \frac{1}{2} \frac{(n-m) ! r^{n-1}}{(n+m) ! r_{0}^{n+2}}\left\{P_{n+1}^{m+1}(\cos \alpha)+(n-m+1)(n-m+2) P_{n+1}^{m-1}(\cos \alpha)\right\}\right. \\
& \times\left\{(n+m)(n+m-1) P_{n-1}^{m-1}(\cos \theta)(\cos m \psi \cos (m-1) \phi+\sin m(m-1) \phi)\right. \\
& \left.+P_{n-1}^{m+1}(\cos \theta)(\cos m \psi \cos (m+1) \phi+\sin m \psi \sin (m+1) \phi\}\right] \\
& d B_{\mathrm{m} y, z}=\frac{d m_{z}}{4 \pi}\left[-\sum_{n=2}^{\infty}(n+1) \frac{r^{n-1}}{r_{0}^{n+2}} P_{n+1}(\cos \alpha) P_{n-1}^{1}(\cos \theta) \sin \phi-\sum_{n=1}^{\infty} \sum_{m=1}^{n} \frac{(n-m) ! r^{n-1}}{(n+m) ! r_{0}^{n+2}}(n-m+1) P_{n+1}^{m}(\cos \alpha)\right. \\
& \times\left\{(n+m)(n+m-1) P_{n-1}^{m-1}(\cos \theta)(\cos m \psi \sin (m-1) \phi-\sin m \psi \cos (m-1) \phi)\right. \\
& \left.-P_{n-1}^{m+1}(\cos \theta)(\cos m \psi \sin (m+1) \phi-\sin m \psi \cos (m+1) \phi\}\right], \\
& d B_{\mathrm{m} z, \rho}=\frac{d m_{\rho}}{4 \pi}\left[\sum_{n=1}^{\infty} n \frac{r^{n-1}}{r_{0}^{n+2}} P_{n+1}^{1}(\cos \alpha) P_{n-1}(\cos \theta)+\sum_{n=2}^{\infty} \sum_{m=1}^{n-1} \frac{(n-m) !}{(n+m-1) !} r_{0}^{n-1}\right. \\
& \left.\times\left\{-(n-m+1) \times(n-m+2) P_{n+1}^{m-1}(\cos \alpha)+P_{n+1}^{m+1}(\cos \alpha)\right\} P_{n-1}^{m}(\cos \theta)(\cos m \psi \cos m \phi+\sin m \psi \sin m \phi)\right],
\end{aligned}
$$




$$
\begin{aligned}
d B_{\mathrm{m} z, \phi}=\frac{d m_{\phi}}{4 \pi}\left[\sum_{n=2}^{\infty} \sum_{m=1}^{n-1} \frac{(n-m) !}{(n+m-1) !} \frac{r^{n-1}}{r_{0}^{n+2}}\left\{(n-m+1)(n-m+2) P_{n+1}^{m-1}(\cos \alpha)+P_{n+1}^{m+1}(\cos \alpha)\right\}\right. \\
\left.\quad \times P_{n-1}^{m}(\cos \theta)(\sin m \psi \cos m \phi-\cos m \psi \sin m \phi)\right], \\
d B_{\mathrm{m} z, z}=\frac{d m_{z}}{4 \pi}\left[\sum_{n=1}^{\infty} n(n+1) \frac{r^{n-1}}{r_{0}^{n+2}} P_{n+1}(\cos \alpha) P_{n-1}(\cos \theta)+\sum_{n=1}^{\infty} \sum_{m=1}^{n-1} 2 \frac{(n-m+1) !}{(n+m-1) !} \frac{r^{n-1}}{r_{0}^{n+2}}\right. \\
\left.\quad \times P_{n+1}^{m}(\cos \alpha) P_{n-1}^{m}(\cos \theta)(\cos m \psi \cos m \phi+\sin m \psi \sin m \phi)\right] .
\end{aligned}
$$

The following relations are also used for obtaining the above equations:

$$
\begin{gathered}
P_{m}^{m}(\cos \theta)=P_{-(n+1)}^{m}(\cos \theta) \\
P_{n}^{-m}(\cos \theta)=(-1)^{m} \frac{(n-m) !}{(n+m) !} P_{n}^{m}(\cos \theta) .
\end{gathered}
$$

We can calculate the magnetic field around the origin generated by the dipole magnetic moment using Eqs. (20)-(31).

\section{B. Magnetic field generated by magnetic moment having volume}

For calculating the detailed magnetic field, the volume of the ferromagnetic material with magnetic moment cannot be sometimes ignored. The dimension of the ferromagnetic material is defined in Fig. 2, and the magnetic moment is supposed to be constant in a piece of ferromagnetic material. The magnetic field $\boldsymbol{B}_{\mathrm{m}}(\boldsymbol{r})$ is obtained by the integration of Eq. (14).

$$
\boldsymbol{B}_{\mathrm{m}}(\boldsymbol{r})=-\int_{z_{1}}^{z_{2}} \int_{\rho_{1}}^{\rho_{2}} \int_{\psi_{1}}^{\psi_{2}} \mu_{0} \nabla_{\mathrm{P}} \Phi \rho \mathrm{d} \psi \mathrm{d} \rho \mathrm{d} z .
$$

Finally, when $r<r_{0}$, we can derive

$$
\begin{aligned}
\boldsymbol{B}_{\mathrm{m}}(\boldsymbol{r})= & \sum_{n=0}^{\infty} \sum_{m=0}^{n}\left[r ^ { n } P _ { n } ^ { m } ( \operatorname { c o s } \theta ) \left\{\mathbf{C}^{\mathrm{m}}(n, m) \cos m \phi\right.\right. \\
& \left.\left.+\boldsymbol{D}^{\mathrm{m}}(n, m) \sin m \phi\right\}\right],
\end{aligned}
$$

where

$$
\begin{aligned}
{\left[\begin{array}{c}
\boldsymbol{C}^{\mathrm{m}}(n, m) \\
\boldsymbol{D}^{\mathrm{m}}(n, m)
\end{array}\right]=} & {\left[\begin{array}{c}
C_{x}^{\mathrm{m}}(n, m) \\
D_{x}^{\mathrm{m}}(n, m)
\end{array}\right] \boldsymbol{i}+\left[\begin{array}{c}
C_{y}^{\mathrm{m}}(n, m) \\
D_{y}^{\mathrm{m}}(n, m)
\end{array}\right] \boldsymbol{j} } \\
& +\left[\begin{array}{c}
C_{z}^{\mathrm{m}}(n, m) \\
D_{z}^{\mathrm{m}}(n, m)
\end{array}\right] \boldsymbol{k} .
\end{aligned}
$$

The coefficients $C^{\mathrm{m}}$ and $D^{\mathrm{m}}$ are calculated from Eqs. (23)-(31) and shown in Eqs. (37)-(49). Equation (49) is calculated by a numerical integration method, such as the Gauss quadrature method.

$$
\left[\begin{array}{c}
C_{x}^{\mathrm{m}}(n, m) \\
D_{x}^{\mathrm{m}}(n, m)
\end{array}\right]=\left[\begin{array}{c}
C_{x, \rho}^{\mathrm{m}} \\
D_{x, \rho}^{\mathrm{m}}
\end{array}\right]+\left[\begin{array}{c}
C_{x, \phi}^{\mathrm{m}} \\
D_{x, \phi}^{\mathrm{m}}
\end{array}\right]+\left[\begin{array}{c}
C_{x, z}^{\mathrm{m}} \\
D_{x, z}^{\mathrm{m}}
\end{array}\right]
$$

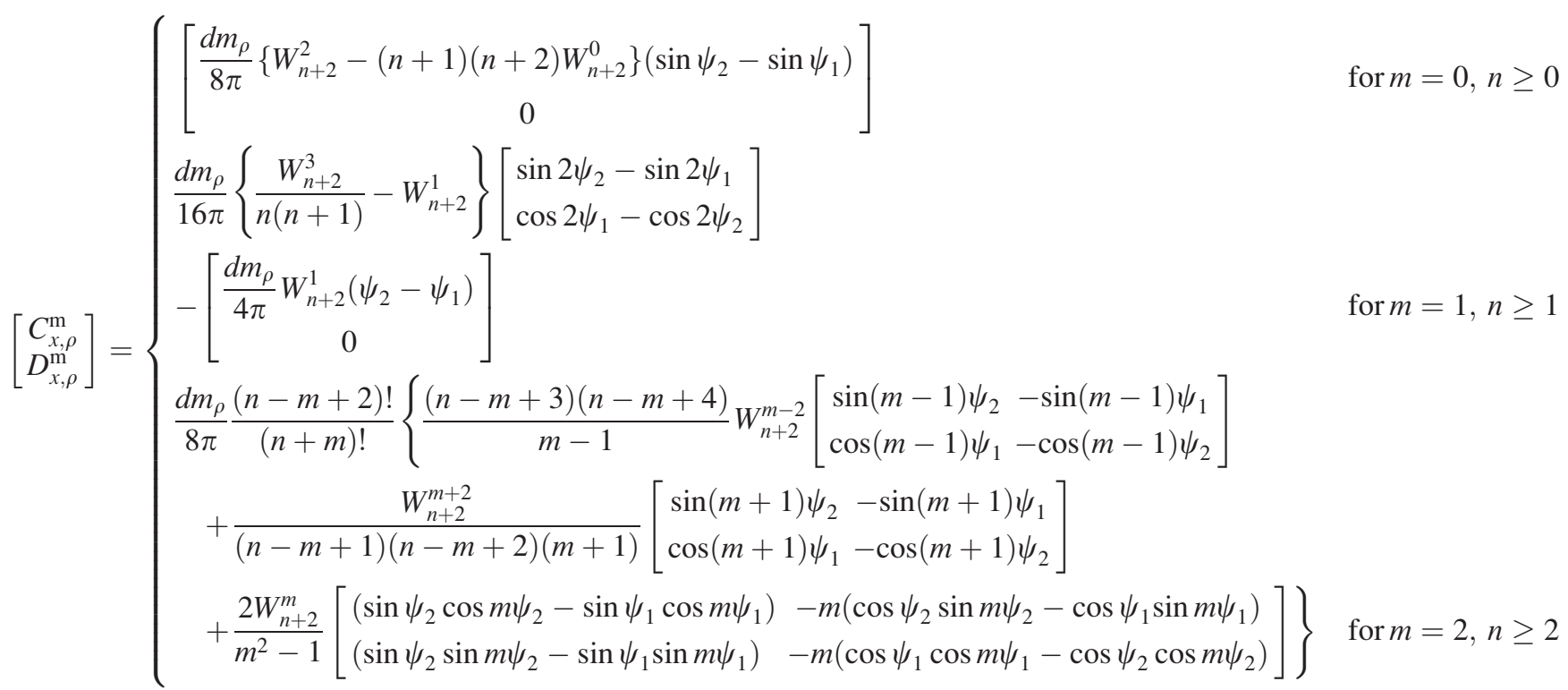




$$
\left[\begin{array}{c}
C_{x, \phi}^{\mathrm{m}} \\
D_{x, \phi}^{\mathrm{m}}
\end{array}\right]=\left\{\begin{array}{cc}
{\left[\begin{array}{l}
-\frac{d m_{\phi}}{8 \pi}\left\{W_{n+2}^{2}+(n+1)(n+2) W_{n+2}^{0}\right\}\left(\cos \psi_{2}-\cos \psi_{1}\right) \\
0
\end{array}\right]} & \text { for } m=0, n \geq 0 \\
-\frac{d m_{\phi}}{16 \pi}\left\{\frac{W_{n+2}^{3}}{n(n+1)}+W_{n+2}^{1}\right\}\left[\begin{array}{c}
\cos 2 \psi_{2}-\cos 2 \psi_{1} \\
\sin 2 \psi_{2}-\sin 2 \psi_{1}
\end{array}\right] & \text { for } m=1, n \geq 1 \\
\frac{d m_{\phi}}{8 \pi} \frac{(n-m+2) !}{(n+m) !}\left\{\frac{(n-m+3)(n-m+4)}{m-1} W_{n+2}^{m-2}\left[\begin{array}{c}
\cos (m-1) \psi_{2}-\cos (m-1) \psi_{1} \\
\sin (m-1) \psi_{2}-\sin (m-1) \psi_{1}
\end{array}\right]\right. & \begin{array}{c}
\cos (m+1) \psi_{2}-\cos (m+1) \psi_{1} \\
-\frac{W_{n+2}^{m+2}}{(n-m+1)(n-m+2)(m+1)}\left[\begin{array}{l}
\sin (m+1) \psi_{2}-\sin (m+1) \psi_{1}
\end{array}\right]
\end{array} \\
\left.+\frac{2 W_{n+2}^{m}}{m^{2}-1}\left[\begin{array}{l}
\left(\cos \psi_{2} \cos m \psi_{2}-\cos \psi_{1} \cos m \psi_{1}\right)-m\left(\sin \psi_{1} \sin m \psi_{1}-\sin \psi_{2} \sin m \psi_{2}\right) \\
\left(\cos \psi_{2} \sin m \psi_{2}-\cos \psi_{1} \sin m \psi_{1}\right)-m\left(\sin \psi_{2} \cos m \psi_{2}-\sin \psi_{1} \cos m \psi_{1}\right)
\end{array}\right]\right\} & \text { for } m=2, n \geq 2
\end{array}\right.
$$

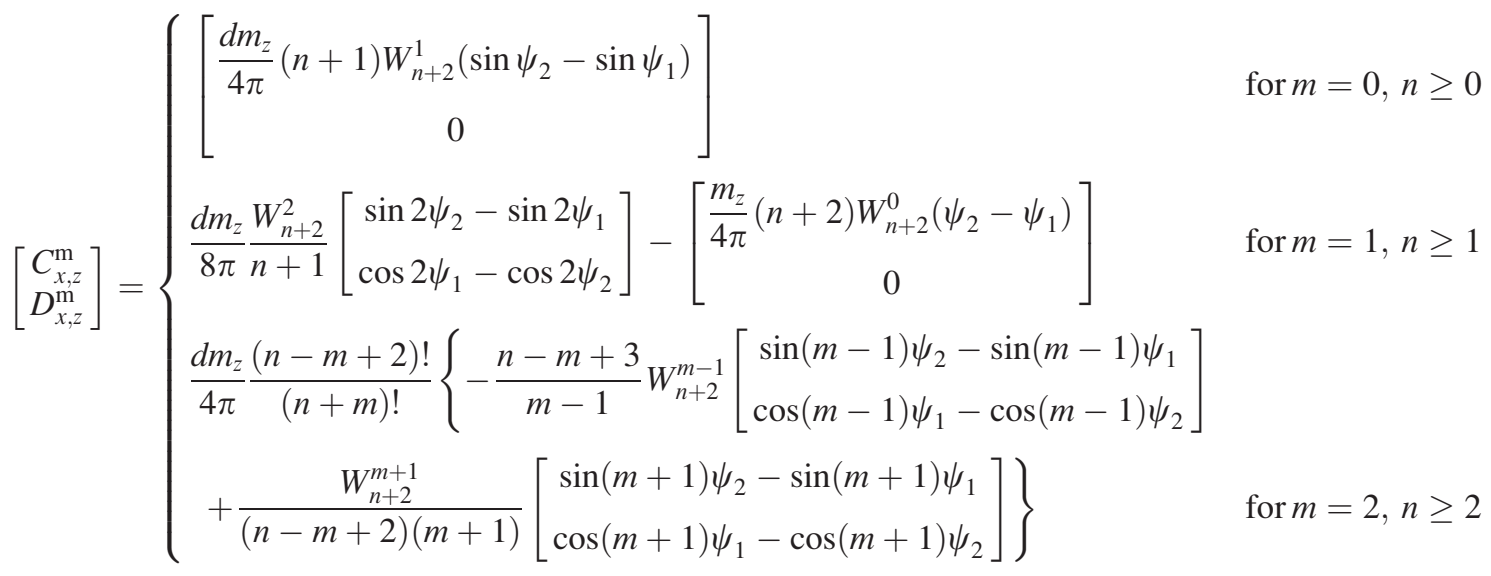

$$
\left[\begin{array}{l}
C_{y}^{\mathrm{m}}(n, m) \\
D_{y}^{\mathrm{m}}(n, m)
\end{array}\right]=\left[\begin{array}{c}
C_{y, \rho}^{\mathrm{m}} \\
D_{y, \rho}^{\mathrm{m}}
\end{array}\right]+\left[\begin{array}{c}
C_{y, \phi}^{\mathrm{m}} \\
D_{y, \phi}^{\mathrm{m}}
\end{array}\right]+\left[\begin{array}{c}
C_{y, z}^{\mathrm{m}} \\
D_{y, z}^{\mathrm{m}}
\end{array}\right]
$$

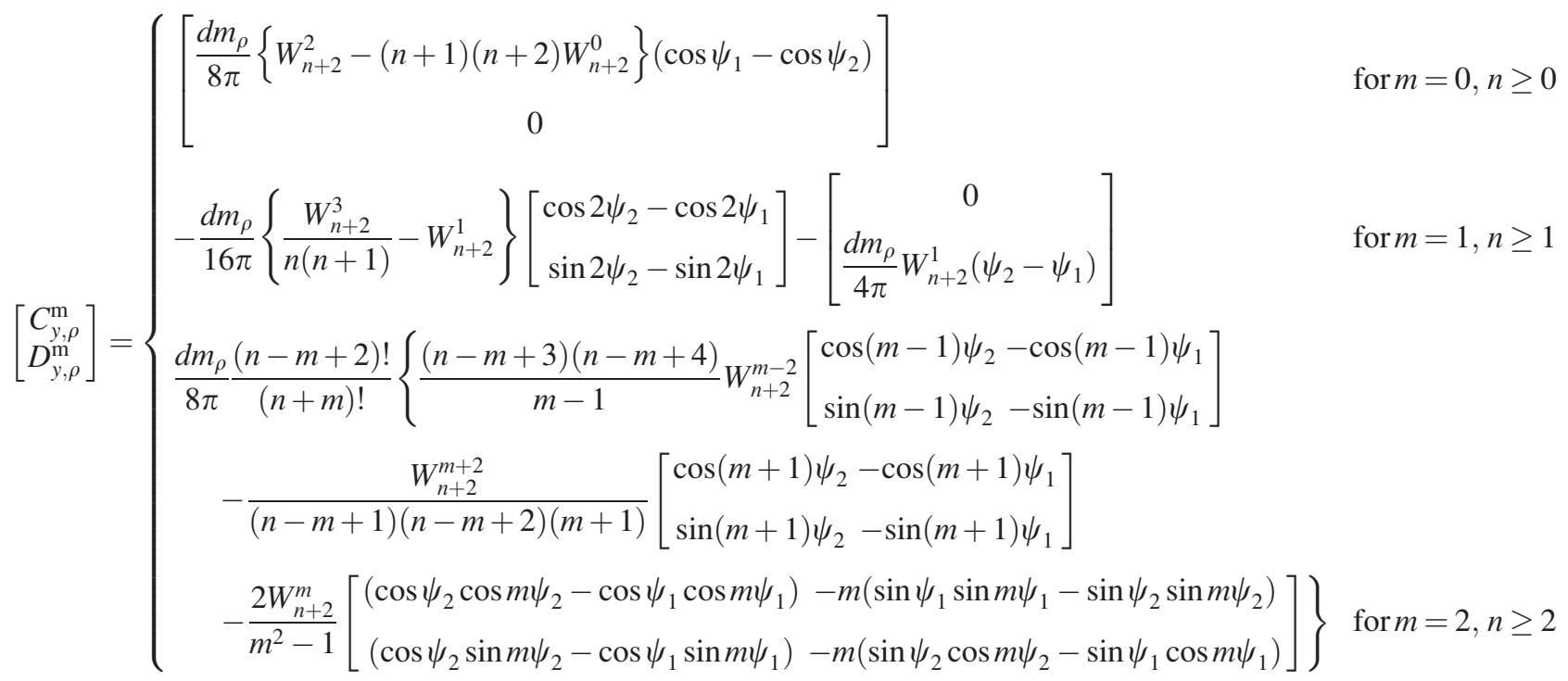




$$
\left[\begin{array}{c}
C_{y, \phi}^{\mathrm{m}} \\
D_{y, \phi}^{\mathrm{m}}
\end{array}\right]=\left\{\begin{array}{cc}
{\left[\begin{array}{l}
-\frac{d m_{\phi}}{8 \pi}\left\{W_{n+2}^{2}+(n+1)(n+2) W_{n+2}^{0}\right\}\left(\sin \psi_{2}-\sin \psi_{1}\right) \\
0
\end{array}\right]} & \text { for } m=0, n \geq 0 \\
-\frac{d m_{\phi}}{16 \pi}\left\{\frac{W_{n+2}^{3}}{n(n+1)}+W_{n+2}^{1}\right\}\left[\begin{array}{l}
\sin 2 \psi_{2}-\sin 2 \psi_{1} \\
\cos 2 \psi_{1}-\cos 2 \psi_{2}
\end{array}\right] & \text { for } m=1, n \geq 1 \\
-\frac{d m_{\phi}}{8 \pi} \frac{(n-m+2) !}{(n+m) !}\left\{\frac{(n-m+3)(n-m+4)}{m-1} W_{n+2}^{m-2}\left[\begin{array}{c}
\sin (m-1) \psi_{2}-\sin (m-1) \psi_{1} \\
\cos (m-1) \psi_{1}-\cos (m-1) \psi_{2}
\end{array}\right]\right. & \\
+\frac{W_{n+2}^{m+2}}{(n-m+1)(n-m+2)(m+1)}\left[\begin{array}{l}
\sin (m+1) \psi_{2}-\sin (m+1) \psi_{1} \\
\cos (m+1) \psi_{1}-\cos (m+1) \psi_{2}
\end{array}\right] & \text { for } m=2, n \geq 2 \\
\left.-\frac{2 W_{n+2}^{m}}{m^{2}-1}\left[\begin{array}{l}
\left(\sin \psi_{2} \cos m \psi_{2}-\sin \psi_{1} \cos m \psi_{1}\right)-m\left(\cos \psi_{2} \sin m \psi_{2}-\cos \psi_{1} \sin m \psi_{1}\right) \\
\left(\sin \psi_{2} \sin m \psi_{2}-\sin \psi_{1} \sin m \psi_{1}\right)-m\left(\cos \psi_{1} \cos m \psi_{1}-\cos \psi_{2} \cos m \psi_{2}\right)
\end{array}\right]\right\} &
\end{array}\right.
$$

$$
\begin{aligned}
& {\left[\begin{array}{c}
C_{y, z}^{\mathrm{m}} \\
D_{y, z}^{\mathrm{m}}
\end{array}\right]=\left\{\begin{array}{cc}
{\left[\begin{array}{c}
-\frac{d m_{z}}{4 \pi}(n+1) W_{n+2}^{1}\left(\cos \psi_{2}-\cos \psi_{1}\right) \\
0
\end{array}\right.} & \text { for } m=0, n \geq 0 \\
-\frac{d m_{z}}{8 \pi} \frac{W_{n+2}^{2}}{n+1}\left[\begin{array}{c}
\cos 2 \psi_{2}-\cos 2 \psi_{1} \\
\sin 2 \psi_{2}-\sin 2 \psi_{1}
\end{array}\right]-\left[\begin{array}{c}
m_{z}(n+2) W_{n+2}^{0}\left(\psi_{2}-\psi_{1}\right) \\
4 \pi
\end{array}\right] & \text { for } m=1, n \geq 1 \\
-\frac{d m_{z}}{4 \pi} \frac{(n-m+2) !}{(n+m) !}\left\{\frac{n-m+3}{m-1} W_{n+2}^{m-1}\left[\begin{array}{c}
\cos (m-1) \psi_{2}-\cos (m-1) \psi_{1} \\
\sin (m-1) \psi_{2}-\sin (m-1) \psi_{1}
\end{array}\right]\right. & \\
\left.+\frac{W_{n+2}^{m+1}}{(n-m+2)(m+1)}\left[\begin{array}{c}
\cos (m+1) \psi_{2}-\cos (m+1) \psi_{1} \\
\sin (m+1) \psi_{2}-\sin (m+1) \psi_{1}
\end{array}\right]\right\} & \text { for } m=2, n \geq 2
\end{array}\right.} \\
& {\left[\begin{array}{c}
C_{z}^{\mathrm{m}}(n, m) \\
D_{z}^{\mathrm{m}}(n, m)
\end{array}\right]=\left[\begin{array}{c}
C_{z, \rho}^{\mathrm{m}} \\
D_{z, \rho}^{\mathrm{m}}
\end{array}\right]+\left[\begin{array}{c}
C_{z, \phi}^{\mathrm{m}} \\
D_{z, \phi}^{\mathrm{m}}
\end{array}\right]+\left[\begin{array}{c}
C_{z, z}^{\mathrm{m}} \\
D_{z, z}^{\mathrm{m}}
\end{array}\right]} \\
& {\left[\begin{array}{c}
C_{z, \rho}^{\mathrm{m}} \\
D_{z, \rho}^{\mathrm{m}}
\end{array}\right]= \begin{cases}{\left[\begin{array}{cc}
\frac{d m_{\rho}}{4 \pi}(n+1) W_{n+2}^{1}\left(\psi_{2}-\psi_{1}\right) \\
0
\end{array}\right]} & \text { for } m=0, n \geq 0 \\
\frac{d m_{\rho}}{4 \pi}\left\{-(n+2) W_{n+2}^{0}+\frac{W_{n+2}^{2}}{n+1}\right\}\left[\begin{array}{c}
\sin \psi_{2}-\sin \psi_{1} \\
\cos \psi_{1}-\cos \psi_{2}
\end{array}\right] & \text { for } m=1, n \geq 1 \\
\frac{d m_{\rho}}{4 \pi m} \frac{(n-m+2) !}{(n+m) !}\left\{-(n-m+3) W_{n+2}^{m-1}+\frac{W_{n+2}^{m+1}}{n-m+2}\right\}\left[\begin{array}{c}
\sin m \psi_{2}-\sin m \psi_{1} \\
\cos m \psi_{1}-\cos m \psi_{2}
\end{array}\right] & \text { for } m=2, n \geq 2\end{cases} } \\
& {\left[\begin{array}{c}
C_{z, \phi}^{\mathrm{m}} \\
D_{z, \phi}^{\mathrm{m}}
\end{array}\right]= \begin{cases}{\left[\begin{array}{l}
0 \\
0
\end{array}\right]} & \text { for } m=0, n \geq 0 \\
-\frac{d m_{\phi}}{4 \pi}\left\{(n+2) W_{n+2}^{0}+\frac{W_{n+2}^{2}}{n+1}\right\}\left[\begin{array}{c}
\cos \psi_{2}-\cos \psi_{1} \\
\sin \psi_{2}-\sin \psi_{1}
\end{array}\right] & \text { for } m=1, n \geq 1 \\
-\frac{d m_{\phi}}{4 \pi m} \frac{(n-m+2) !}{(n+m) !}\left\{(n-m+3) W_{n+2}^{m-1}+\frac{W_{n+2}^{m+1}}{n-m+2}\right\}\left[\begin{array}{c}
\cos m \psi_{2}-\cos m \psi_{1} \\
\sin m \psi_{2}-\sin m \psi_{1}
\end{array}\right] & \text { for } m=2, n \geq 2\end{cases} }
\end{aligned}
$$




$$
\left[\begin{array}{c}
C_{z, z}^{\mathrm{m}} \\
D_{z, z}^{\mathrm{m}}
\end{array}\right]=\left\{\begin{array}{cc}
{\left[\begin{array}{cc}
\frac{d m_{z}}{4 \pi}(n+1)(n+2) W_{n+2}^{0}\left(\psi_{2}-\psi_{1}\right) \\
0
\end{array}\right]} & \text { for } m=0, n \geq 0 \\
\frac{d m_{z}}{2 \pi} W_{n+2}^{1}\left[\begin{array}{c}
\sin \psi_{2}-\sin \psi_{1} \\
\cos \psi_{1}-\cos \psi_{2}
\end{array}\right] & \text { for } m=1, n \geq 1 \\
\frac{d m_{z}}{2 \pi m} \frac{(n-m+2) !}{(n+m) !} W_{n+2}^{m}\left[\begin{array}{c}
\sin m \psi_{2}-\sin m \psi_{1} \\
\cos m \psi_{1}-\cos m \psi_{2}
\end{array}\right] & \text { for } m=2, n \geq 2 \\
W_{n}^{m} \equiv \int_{z_{1}}^{z_{2}} \int_{\rho_{1}}^{\rho_{2}} \frac{1}{r_{0}^{n+1} P_{n}^{m}(\cos \alpha) \rho \mathrm{d} \rho \mathrm{d} z .} &
\end{array}\right.
$$

\section{Magnetic field generated by current}

The magnetic field $\boldsymbol{B}_{\mathrm{c}}(\boldsymbol{r})$ generated by current can be also represented with the spherical harmonic functions.

A line current $\boldsymbol{d} \boldsymbol{I}$ shown in Fig. 1 generates the magnet field

$$
\boldsymbol{B}_{\mathrm{c}}(\boldsymbol{r})=\frac{\mu_{0}}{4 \pi} \int_{l} \boldsymbol{d I} \times \nabla_{\mathrm{Q}}\left(\frac{1}{R}\right) \mathrm{d} l,
$$

where $l$ is the path of the line current.

\section{Circular line current}

When a circular line current (one-turn coil), whose axis corresponds to the $z$-axis, is considered as shown in Fig. 3, the magnetic field $\boldsymbol{B}_{\mathrm{c}}(\boldsymbol{r})$ is given as

$$
\boldsymbol{B}_{\mathrm{c}}(\boldsymbol{r})=\frac{\mu_{0}}{4 \pi} \int_{0}^{2 \pi} F_{n m} \sum_{n=0}^{\infty} \sum_{m=0}^{n} r^{n} P_{n}^{m}(\cos \theta) \rho I_{\phi}\left(\cos \psi \frac{\partial T_{n m}^{\prime}}{\partial z}, \sin \psi \frac{\partial T_{n m}^{\prime}}{\partial z},-\cos \psi \frac{\partial T_{n m}^{\prime}}{\partial x}-\sin \psi \frac{\partial T_{n m}^{\prime}}{\partial y}\right) \mathrm{d} \psi
$$

where $I_{\phi}$ is the current of the circular coil, when $r<r_{0}$. The magnetic field can be also expressed with the spherical harmonic functions, as follows:

$$
\boldsymbol{B}_{\mathrm{c}}(\boldsymbol{r})=\sum_{n=0}^{\infty} \sum_{m=0}^{n}\left[r^{n} P_{n}^{m}(\cos \theta)\left\{\boldsymbol{C}^{\mathrm{c}}(n, m) \cos m \phi+\boldsymbol{D}^{\mathrm{c}}(n, m) \sin m \phi\right\}\right],
$$

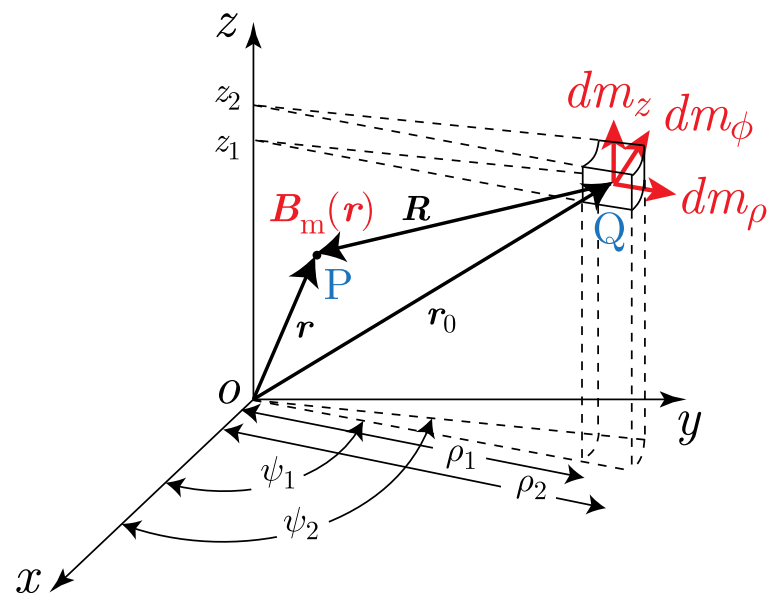

FIG. 2. Magnetic field $\boldsymbol{B}_{\mathrm{m}}(\boldsymbol{r})$ at point $\mathrm{P}$ is generated by ferromagnetic material having volume with magnetic moment $\boldsymbol{d} \boldsymbol{m}$ at point $\mathrm{Q}$. The magnetic moment $\boldsymbol{d} \boldsymbol{m}=\left(d m_{\rho}, d m_{\phi}, d m_{z}\right)$ is supposed to be constant in a small piece of ferromagnetic material.

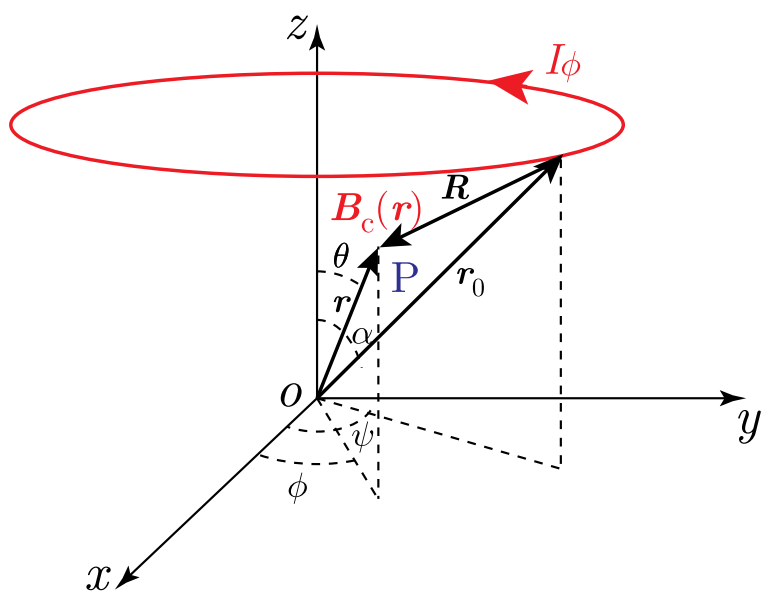

FIG. 3. Magnetic field $\boldsymbol{B}_{\mathrm{c}}(\boldsymbol{r})$ at point $\mathrm{P}$ is generated by circular coil current $I_{\phi}$ (one-turn coil). The axis of the circular coil is identical to the $z$-axis. 
where

$$
\begin{aligned}
{\left[\begin{array}{l}
\boldsymbol{C}^{\mathrm{c}}(n, m) \\
\boldsymbol{D}^{\mathrm{c}}(n, m)
\end{array}\right]=} & {\left[\begin{array}{l}
C_{x}^{\mathrm{c}}(n, m) \\
D_{x}^{\mathrm{c}}(n, m)
\end{array}\right] \boldsymbol{i}+\left[\begin{array}{c}
C_{y}^{\mathrm{c}}(n, m) \\
D_{y}^{\mathrm{c}}(n, m)
\end{array}\right] \boldsymbol{j} } \\
& +\left[\begin{array}{c}
C_{z}^{\mathrm{c}}(n, m) \\
D_{z}^{\mathrm{c}}(n, m)
\end{array}\right] \boldsymbol{k} .
\end{aligned}
$$

The coefficients $C^{\mathrm{c}}$ and $D^{\mathrm{c}}$ are calculated by the integration of $\psi$ and given in Eqs. (54)-(56).

$$
\left[\begin{array}{l}
C_{x}^{\mathrm{c}}(n, m) \\
D_{x}^{\mathrm{c}}(n, m)
\end{array}\right]= \begin{cases}{\left[\begin{array}{ll}
-\frac{\mu_{0}}{2} \frac{I_{\phi}}{n+1} \frac{\sin \alpha}{r_{0}^{n+1}} P_{n+1}^{1}(\cos \alpha) \\
0
\end{array}\right]} & \\
{\left[\begin{array}{l}
0 \\
0
\end{array}\right]} & \text { for } m=1\end{cases}
$$

$$
\left[\begin{array}{l}
C_{y}^{\mathrm{c}}(n, m) \\
D_{y}^{\mathrm{c}}(n, m)
\end{array}\right]=\left\{\begin{array}{cc}
{\left[\begin{array}{cc}
0 \\
-\frac{\mu_{0}}{2} \frac{I_{\phi}}{n+1} \frac{\sin \alpha}{r_{0}^{n+1}} P_{n+1}^{1}(\cos \alpha)
\end{array}\right]} \\
{\left[\begin{array}{l}
0 \\
0
\end{array}\right]} & \text { for } m=1 \\
& \text { for } m \neq 1
\end{array}\right.
$$

$$
\left[\begin{array}{l}
C_{z}^{\mathrm{c}}(n, m) \\
D_{z}^{\mathrm{c}}(n, m)
\end{array}\right]= \begin{cases}{\left[\begin{array}{cc}
\frac{\mu_{0}}{2} I_{\phi} \frac{\sin \alpha}{r_{0}^{n+1}} P_{n+1}^{1}(\cos \alpha) \\
0
\end{array}\right]} & \text { for } m=0 \\
{\left[\begin{array}{l}
0 \\
0
\end{array}\right]} & \text { for } m \neq 0 .\end{cases}
$$

\section{Solenoid coil}

A magnetic field generated by a solenoid coil with rectangular cross section can be obtained from the integration of Eq. (51), as follows:

$$
\begin{aligned}
\boldsymbol{B}_{\mathrm{c}}(\boldsymbol{r})= & \frac{\mu_{0}}{4 \pi} \int_{b_{1}}^{b_{2}} \int_{a_{1}}^{a_{2}} \int_{0}^{2 \pi} F_{n m} \sum_{n=0}^{\infty} \sum_{m=0}^{n} r^{n} P_{n}^{m}(\cos \theta) \rho j \\
& \times\left(\cos \psi \frac{\partial T_{n m}^{\prime}}{\partial z}, \sin \psi \frac{\partial T_{n m}^{\prime}}{\partial z},-\cos \psi \frac{\partial T_{n m}^{\prime}}{\partial x}\right. \\
& \left.-\sin \psi \frac{\partial T_{n m}^{\prime}}{\partial y}\right) \mathrm{d} \psi \mathrm{d} \rho \mathrm{d} z,
\end{aligned}
$$

where $j, a_{1}, a_{2}, b_{1}$, and $b_{2}$ are the current density, the inner radius, the outer radius, the bottom surface position, and the top surface position of the solenoid coil, respectively, as

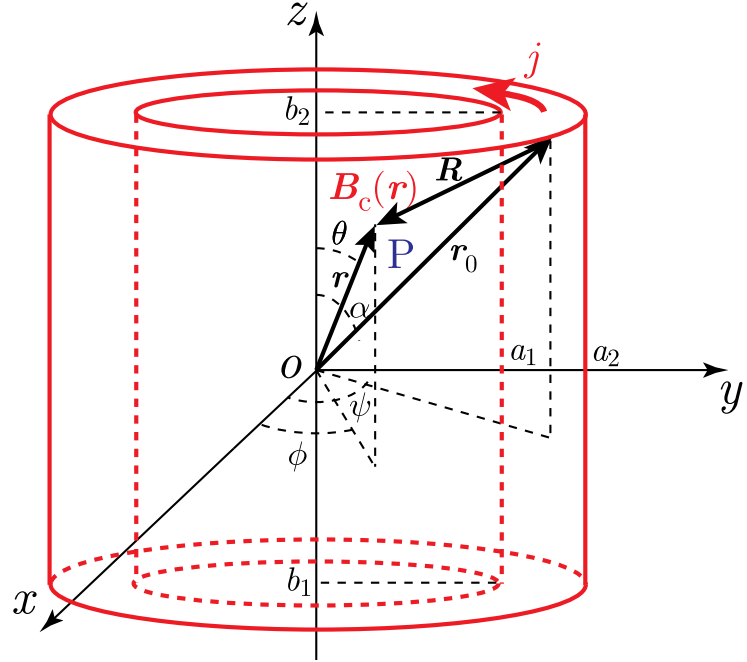

FIG. 4. Solenoid coil with constant current density $j$ generates magnetic field $\boldsymbol{B}_{\mathrm{c}}(\boldsymbol{r})$. The inner and outer radii are $a_{1}$ and $a_{2}$. The bottom and top surfaces of the coil are $b_{1}$ and $b_{2}$.

shown in Fig. 4. The current density $j$ is supposed to be constant everywhere in the solenoid coil. The magnetic field expression with the spherical harmonic functions is, similarly to the circular coil, given as

where

$$
\begin{aligned}
\boldsymbol{B}_{\mathrm{c}}(\boldsymbol{r})= & \sum_{n=0}^{\infty} \sum_{m=0}^{n}\left[r ^ { n } P _ { n } ^ { m } ( \operatorname { c o s } \theta ) \left\{\boldsymbol{C}^{\mathrm{c}}(n, m) \cos m \phi\right.\right. \\
& \left.\left.+\boldsymbol{D}^{\mathrm{c}}(n, m) \sin m \phi\right\}\right],
\end{aligned}
$$

$$
\begin{aligned}
{\left[\begin{array}{l}
\boldsymbol{C}^{\mathrm{c}}(n, m) \\
\boldsymbol{D}^{\mathrm{c}}(n, m)
\end{array}\right]=} & {\left[\begin{array}{l}
C_{x}^{\mathrm{c}}(n, m) \\
D_{x}^{\mathrm{c}}(n, m)
\end{array}\right] \boldsymbol{i}+\left[\begin{array}{l}
C_{y}^{\mathrm{c}}(n, m) \\
D_{y}^{\mathrm{c}}(n, m)
\end{array}\right] \boldsymbol{j} } \\
& +\left[\begin{array}{c}
C_{z}^{\mathrm{c}}(n, m) \\
D_{z}^{\mathrm{c}}(n, m)
\end{array}\right] \boldsymbol{k}
\end{aligned}
$$

$\left[\begin{array}{l}C_{x}^{\mathrm{c}}(n, m) \\ D_{x}^{\mathrm{c}}(n, m)\end{array}\right]= \begin{cases}{\left[\begin{array}{cc}-\frac{\mu_{0}}{2} \frac{j}{n+1} U_{n+1} \\ 0\end{array}\right]} & \text { for } m=1 \\ {\left[\begin{array}{l}0 \\ 0\end{array}\right]} & \text { for } m \neq 1\end{cases}$

$$
\left[\begin{array}{c}
C_{y}^{\mathrm{c}}(n, m) \\
D_{y}^{\mathrm{c}}(n, m)
\end{array}\right]= \begin{cases}{\left[\begin{array}{c}
0 \\
-\frac{\mu_{0}}{2} \frac{j}{n+1} U_{n+1}
\end{array}\right]} & \text { for } m=1 \\
{\left[\begin{array}{l}
0 \\
0
\end{array}\right]} & \text { for } m \neq 1\end{cases}
$$

$$
\left[\begin{array}{c}
C_{z}^{\mathrm{c}}(n, m) \\
D_{z}^{\mathrm{c}}(n, m)
\end{array}\right]= \begin{cases}{\left[\begin{array}{cc}
\frac{\mu_{0}}{2} j U_{n+1} \\
0
\end{array}\right]} & \text { for } m=0 \\
{\left[\begin{array}{l}
0 \\
0
\end{array}\right]} & \text { for } m \neq 0\end{cases}
$$




$$
U_{n+1}=\int_{b_{1}}^{b_{2}} \int_{a_{1}}^{a_{2}} \frac{\sin \alpha}{r_{0}^{n+1}} P_{n+1}^{1}(\cos \alpha) \mathrm{d} \rho \mathrm{d} z .
$$

When the solenoid coil is placed at the origin $\left(b_{1}=-b_{2}\right)$, the expression can be simplified. Due to the symmetry, the coefficients $C^{c}$ and $D^{c}$ are 0 when $n$ is odd. The coefficient $U_{n+1}$ is represented using the Fabry factor $G(\gamma, \eta)$, where $\gamma=a_{2} / a_{1}$ and $\eta=b_{2} / a_{1}{ }^{20}$

$$
\begin{aligned}
U_{n+1}=\left\{\begin{array}{cc}
\frac{5 a_{1}}{\pi} G(\gamma, \eta) E_{n} & \text { if } n \text { is even } \\
0 & \text { if } n \text { is odd }
\end{array}\right. \\
G(\gamma, \eta)=\frac{4 \pi}{10} \eta \ln \frac{\gamma+\sqrt{\gamma^{2}+\eta^{2}}}{1+\sqrt{1+\eta^{2}}} \\
=\frac{4 \pi}{10} \eta\left\{\sinh ^{-1} \frac{\gamma}{\eta}-\sinh ^{-1} \frac{1}{\eta}\right\},
\end{aligned}
$$

where $E_{n}$ is the coefficient of the Taylor expansion at the magnet center.

$$
E_{n}=\frac{1}{B_{0}} \frac{1}{n !}\left[\frac{\mathrm{d}^{n} B_{z}(z, 0)}{\mathrm{d} z^{n}}\right]_{z=0},
$$

where $B_{0}$ and $B_{z}(z, 0)$ are the central magnetic field and the magnetic field along the $z$-axis, respectively.

$$
\begin{aligned}
& B_{0}=\mu_{0} j a_{1} \eta \ln \frac{\gamma+\sqrt{\gamma^{2}+\eta^{2}}}{1+\sqrt{1+\eta^{2}}}=10^{-6} \times j a_{1} G(\gamma, \eta) \\
& B_{z}(z, 0)=\frac{\mu_{0} j}{2}\left[\left(b_{2}+z\right) \ln \frac{a_{2}+\sqrt{a_{2}^{2}+\left(b_{2}+z\right)^{2}}}{a_{1}+\sqrt{a_{1}^{2}+\left(b_{2}+z\right)^{2}}}\right. \\
& \left.+\left(b_{2}-z\right) \ln \frac{a_{2}+\sqrt{a_{2}^{2}+\left(b_{2}-z\right)^{2}}}{a_{1}+\sqrt{a_{1}^{2}+\left(b_{2}-z\right)^{2}}}\right] \text {. }
\end{aligned}
$$

The simplified expression of $G(\gamma, \eta) E_{n}$ is shown in Appendix C.

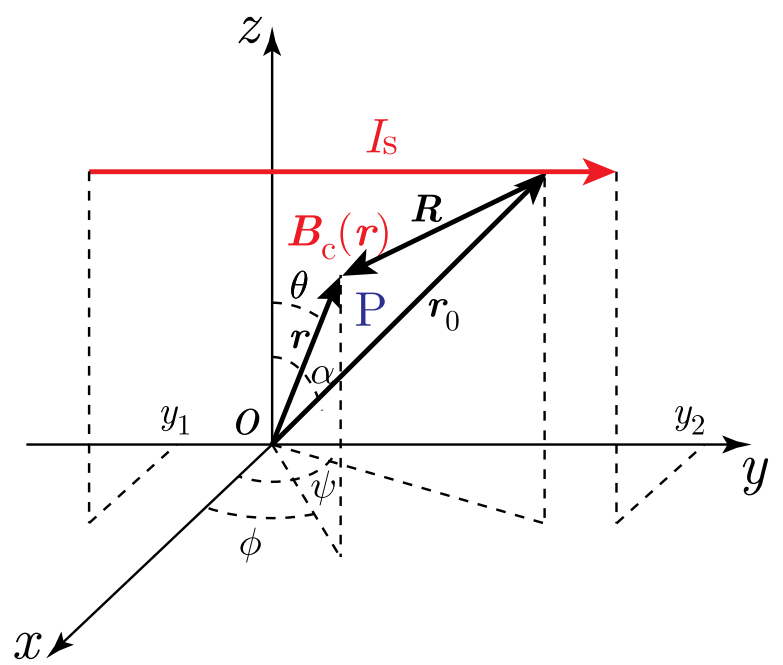

FIG. 5. Magnetic field $\boldsymbol{B}_{\mathrm{c}}(\boldsymbol{r})$ at point $\mathrm{P}$ is generated by straight current $I_{\mathrm{s}}$ from $y_{1}$ to $y_{2}$. The current is parallel to the $y$-axis.

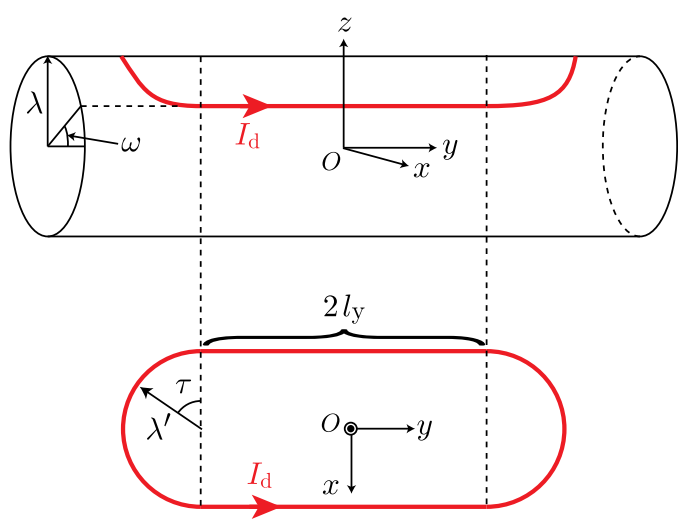

FIG. 6. A dipole coils sticking on a tube with radius of $\lambda$. The length of straight section is $2 l_{\mathrm{y}}$.

\section{Straight current}

A magnetic field $\boldsymbol{B}_{\mathrm{c}}(\boldsymbol{r})$ generated by a straight current parallel to $y$-axis, as shown in Fig. 5, is as follows:

$$
\begin{aligned}
\boldsymbol{B}_{\mathrm{c}}(\boldsymbol{r})= & \frac{\mu_{0}}{4 \pi} \int_{y_{1}}^{y_{2}} F_{n m} \sum_{n=0}^{\infty} \sum_{m=0}^{n} r^{n} P_{n}^{m}(\cos \theta) I_{\mathrm{s}} \\
& \times\left(\frac{\partial T_{n m}^{\prime}}{\partial z}, 0,-\frac{\partial T_{n m}^{\prime}}{\partial x}\right) \mathrm{d} y,
\end{aligned}
$$

where $I_{\mathrm{s}}$ is the current of the straight current from $y_{1}$ to $y_{2}$, when $r<r_{0}$. The magnetic field represented with the spherical harmonic functions is

$$
\begin{aligned}
\boldsymbol{B}_{\mathrm{c}}(\boldsymbol{r})= & \sum_{n=0}^{\infty} \sum_{m=0}^{n}\left[r ^ { n } P _ { n } ^ { m } ( \operatorname { c o s } \theta ) \left\{\boldsymbol{C}^{\mathrm{s}}(n, m) \cos m \phi\right.\right. \\
& \left.\left.+\boldsymbol{D}^{\mathrm{s}}(n, m) \sin m \phi\right\}\right],
\end{aligned}
$$

\begin{tabular}{|c|c|c|c|c|c|}
\hline & & \multicolumn{4}{|c|}{$m$} \\
\hline & & 0 & 1 & 2 & 3 \\
\hline \multirow[t]{4}{*}{$n$} & 0 & $-2.50 \times 10^{-5}$ & $\ldots$ & $\ldots$ & $\ldots$ \\
\hline & 1 & $5.94 \times 10^{-6}$ & $-2.44 \times 10^{-4}$ & $\ldots$ & $\ldots$ \\
\hline & 2 & $-1.19 \times 10^{-3}$ & $-1.74 \times 10^{-4}$ & $7.13 \times 10^{-3}$ & $\ldots$ \\
\hline & 3 & $-8.47 \times 10^{-4}$ & $3.47 \times 10^{-2}$ & $8.47 \times 10^{-3}$ & $-3.48 \times 10^{-1}$ \\
\hline
\end{tabular}

where

$$
\left[\begin{array}{l}
\boldsymbol{C}^{\mathrm{s}}(n, m) \\
\boldsymbol{D}^{\mathrm{s}}(n, m)
\end{array}\right]=\left[\begin{array}{c}
C_{x}^{\mathrm{s}}(n, m) \\
D_{x}^{\mathrm{s}}(n, m)
\end{array}\right] \boldsymbol{i}+\left[\begin{array}{c}
C_{z}^{\mathrm{s}}(n, m) \\
D_{z}^{\mathrm{s}}(n, m)
\end{array}\right] \boldsymbol{k} .
$$

The coefficients $C^{\mathrm{s}}$ and $D^{\mathrm{s}}$, with the integration from $y_{1}$ to $y_{2}$, are given by

$$
\begin{aligned}
{\left[\begin{array}{c}
C_{x}^{\mathrm{s}}(n, m) \\
D_{x}^{\mathrm{s}}(n, m)
\end{array}\right]=} & -\frac{\mu_{0}}{4 \pi} F_{n m}(n-m+1) I_{\mathrm{s}} \int_{y_{1}}^{y_{2}} \frac{P_{n+1}^{m}(\cos \alpha)}{r_{0}^{n+2}} \\
& \times\left[\begin{array}{c}
\cos m \psi \\
\sin m \psi
\end{array}\right] \mathrm{d} y
\end{aligned}
$$

TABLE I. Numerical integration value of $W_{n}^{m}$ with 200 evaluation points $\left(\rho_{1}=100 \mathrm{~mm}, \rho_{2}=105 \mathrm{~mm}, z_{1}=0 \mathrm{~mm}, z_{2}=5 \mathrm{~mm}\right)$. 


$$
\left[\begin{array}{c}
C_{z}^{\mathrm{s}}(n, m) \\
D_{z}^{\mathrm{s}}(n, m)
\end{array}\right]=\frac{\mu_{0}}{8 \pi} F_{n m} I_{\mathrm{s}} \int_{y_{1}}^{y_{2}} \frac{1}{r_{0}^{n+2}}\left\{-(n-m+1)(n-m+2) P_{n+1}^{m-1}(\cos \alpha)\left[\begin{array}{c}
\cos (m-1) \psi \\
\sin (m-1) \psi
\end{array}\right]+P_{n+1}^{m+1}(\cos \alpha)\left[\begin{array}{c}
\cos (m+1) \psi \\
\sin (m+1) \psi
\end{array}\right]\right\} \mathrm{d} y .
$$

To calculate these equations, the numerical integration is required.

\section{Dipole coil current}

Let us consider a dipole coil as shown in Fig. 6. The locus $(x, y, z)$ of the curve section is represented by

$$
\begin{gathered}
x=\lambda \sin \left(\frac{\lambda^{\prime} \cos \tau}{\lambda}\right) \\
y=\lambda^{\prime} \sin \tau \pm l_{\mathrm{y}} \\
z=\lambda \cos \left(\frac{\lambda^{\prime} \cos \tau}{\lambda}\right) \\
\lambda^{\prime}=\left(\frac{\pi}{2}-\omega\right) \lambda,
\end{gathered}
$$

where $\lambda$ is the radius of the tube, $l_{\mathrm{y}}$ is the half length of straight section, and $\tau$ and $\omega$ are the angles shown in Fig. 6.

The coefficients of the spherical harmonics functions with respect to the straight section are shown by Eqs. (72) and (73). When $y_{1}=-l_{\mathrm{y}}$ and $y_{2}=l_{\mathrm{y}}$, the coefficients $D^{\mathrm{s}}$ in Eqs. (72) and (73) become zero.

On the other hand, it is difficult to calculate the coefficients of the spherical harmonics functions on the curve section. Let the current vector be $I_{\mathrm{d}}=\left(I_{\mathrm{d} x}, I_{\mathrm{d} y}, I_{\mathrm{d} z}\right)$ on the curve section. The orientation of the current vector can be derived from the differential of Eqs. (74)-(76).

$$
\begin{gathered}
\frac{\mathrm{d} x}{\mathrm{~d} \tau}=-\lambda^{\prime} \cos \left(\frac{\lambda^{\prime} \cos \tau}{\lambda}\right) \sin \tau \\
\frac{\mathrm{d} y}{\mathrm{~d} \tau}=\lambda^{\prime} \cos \tau \\
\frac{\mathrm{d} z}{\mathrm{~d} \tau}=\lambda^{\prime} \sin \left(\frac{\lambda^{\prime} \cos \tau}{\lambda}\right) \sin \tau .
\end{gathered}
$$

Therefore, we can obtain

$$
\begin{gathered}
I_{\mathrm{d} x}=I_{\mathrm{d}} \frac{\mathrm{d} x}{\mathrm{~d} \tau} / u \\
I_{\mathrm{d} y}=I_{\mathrm{d}} \frac{\mathrm{d} y}{\mathrm{~d} \tau} / u \\
I_{\mathrm{d} z}=I_{\mathrm{d}} \frac{\mathrm{d} z}{\mathrm{~d} \tau} / u \\
u=\sqrt{\left(\frac{\mathrm{d} x}{\mathrm{~d} \tau}\right)^{2}+\left(\frac{\mathrm{d} y}{\mathrm{~d} \tau}\right)^{2}+\left(\frac{\mathrm{d} z}{\mathrm{~d} \tau}\right)^{2}} .
\end{gathered}
$$

\begin{tabular}{|c|c|c|c|c|c|}
\hline & & \multicolumn{4}{|c|}{$m$} \\
\hline & & 0 & 1 & 2 & 3 \\
\hline \multirow[t]{4}{*}{$n$} & 0 & $1.77 \times 10^{-5}$ & $\ldots$ & $\ldots$ & $\ldots$ \\
\hline & 1 & $8.62 \times 10^{-5}$ & $-8.62 \times 10^{-5}$ & $\ldots$ & $\ldots$ \\
\hline & 2 & $2.10 \times 10^{-4}$ & $-1.26 \times 10^{-3}$ & $1.26 \times 10^{-3}$ & $\ldots$ \\
\hline & 3 & $-1.03 \times 10^{-3}$ & $-9.23 \times 10^{-3}$ & $3.08 \times 10^{-2}$ & $-3.08 \times 10^{-2}$ \\
\hline
\end{tabular}

Finally, the magnetic field $\boldsymbol{B}_{\mathrm{c}}(\boldsymbol{r})$ on the curve sections of the dipole coil is represented by

$$
\boldsymbol{B}_{\mathrm{c}}(\boldsymbol{r})=\frac{\mu_{0}}{4 \pi} \int_{0}^{2 \pi} F_{n m} \sum_{n=0}^{\infty} \sum_{m=0}^{n} r^{n} P_{n}^{m}(\cos \theta)\left(I_{\mathrm{d} y} \frac{\partial T_{n m}^{\prime}}{\partial z}-I_{\mathrm{d} z} \frac{\partial T_{n m}^{\prime}}{\partial y}, I_{\mathrm{d} z} \frac{\partial T_{n m}^{\prime}}{\partial x}-I_{\mathrm{d} x} \frac{\partial T_{n m}^{\prime}}{\partial z}, I_{\mathrm{d} x} \frac{\partial T_{n m}^{\prime}}{\partial y}-I_{\mathrm{d} y} \frac{\partial T_{n m}^{\prime}}{\partial x}\right) u \mathrm{~d} \tau
$$

using Eqs. (81)-(83) and (74)-(76) for the calculation of $r_{0}$. From Eq. (85), it is possible to compute the spherical harmonics coefficient of the curve section of the dipole coil by means of the numerical integration.

TABLE II. Numerical integration value of $W_{n}^{m}$ with 200 evaluation points ( $\left.\rho_{1}=100 \mathrm{~mm}, \rho_{2}=105 \mathrm{~mm}, z_{1}=100 \mathrm{~mm}, z_{2}=105 \mathrm{~mm}\right)$.

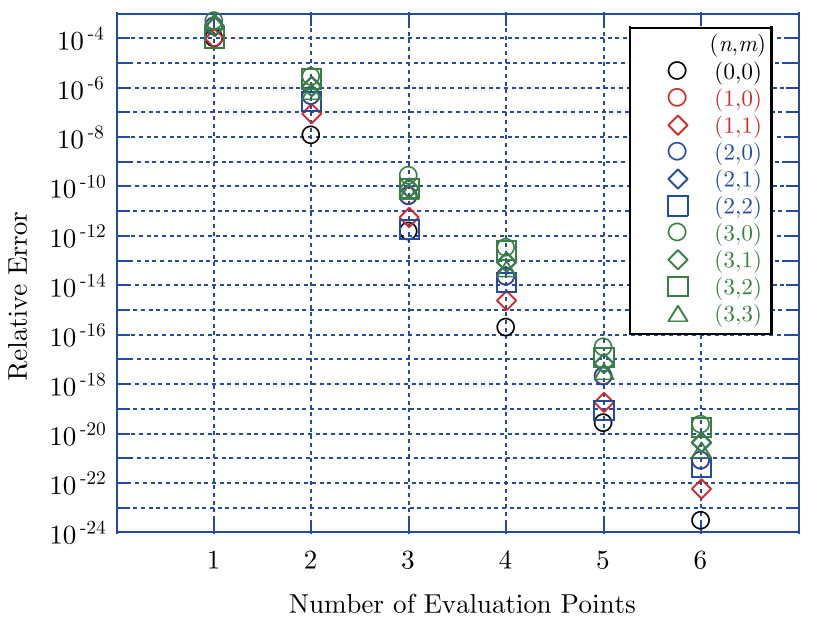

FIG. 7. Relative error of numerical integration $W_{n}^{m}$ of Eq. (49) in the range of $\rho_{1}=100$ to $\rho_{2}=105 \mathrm{~mm}$ and $z_{1}=0$ to $z_{2}=5 \mathrm{~mm}$ when the number of evaluation points is changed. The solution with 200 evaluation points is the standard of comparison. 


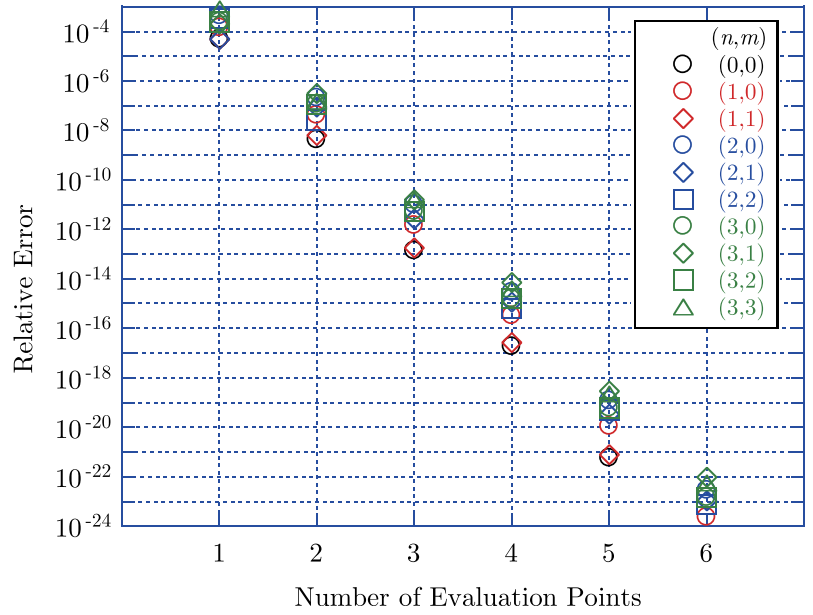

FIG. 8. Relative error of numerical integration $W_{n}^{m}$ of Eq. (49) in the range of $\rho_{1}=100$ to $\rho_{2}=105 \mathrm{~mm}$ and $z_{1}=0$ to $z_{2}=5 \mathrm{~mm}$. when the number of evaluation points is changed. The solution with 200 evaluation points is the standard of comparison.

\section{SPHERICAL HARMONIC COEFFICIENT CALCULATION}

The calculation of the above-given spherical harmonic coefficients needs a numerical integration. It is necessary to check the accuracy of the coefficient calculation with the numeral integration. In this chapter, the accuracy of the numerical integration results is investigated for a magnetized ferromagnetic material piece, a circular coil with small and

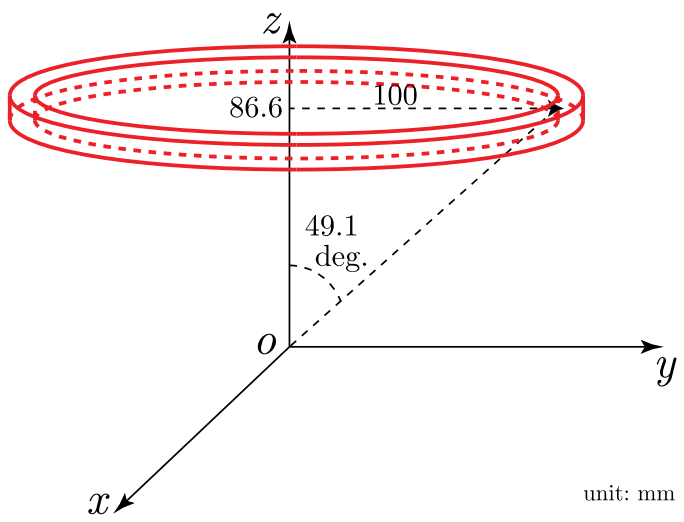

(a)

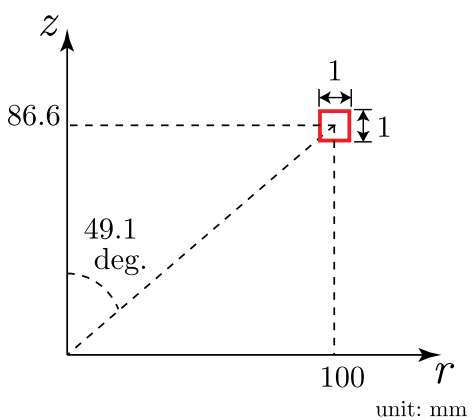

(b)

FIG. 9. A circular coil with small cross section as a simple test model. (a) Schematic view and (b) cross-sectional view.
TABLE III. Spherical harmonic coefficients of ring coil with small cross section.

\begin{tabular}{cccc}
\hline \hline Order $n$ & 0 & 1 & 2 \\
$C_{z}^{\mathrm{c}}(n, 0)$ & $2.713 \times 10^{-6}$ & $4.028 \times 10^{-5}$ & $2.659 \times 10^{-4}$ \\
Order $n$ & 3 & 4 & 5 \\
$C_{z}^{\mathrm{c}}(n, 0)$ & $1.504 \times 10^{-6}$ & $1.897 \times 10^{-2}$ & $2.066 \times 10^{-1}$ \\
\hline \hline
\end{tabular}

large cross section, a straight line current, and a dipole coil. Since this research aims the shimming of MRI or NMR magnet system, it is supposed that the bore of coils or ferromagnetic material is relatively small and $r<r_{0}$.

In this paper, the Gauss quadrature method is employed as the numerical integration.

\section{A. Piece of magnetized ferromagnetic material}

It is necessary to numerically integrate $W_{n}^{m}$ of Eq. (49) for calculation of the spherical harmonic coefficients of the magnetic field contributed by a ferromagnetic material piece with magnetic moment. The accuracy of the spherical harmonics coefficients depends on the number of evaluation points for the numerical integration in the radial and $z$-axial direction. In this paper, $1 \times 1$ to $6 \times 6$ evaluation points are examined in order to evaluate the relative error. 200 evaluation points are regarded as the standard. The integration range is from 100.0 to $105.0 \mathrm{~mm}$ in the radial direction and from 0.0 to $5.0 \mathrm{~mm}$ and from 100.0 to $105.0 \mathrm{~mm}$ in the $z$-direction.

Tables I and II show the results of the numerical integration $W_{n}^{m}$ when 200 evaluation points are adopted as the standard in the Gaussian quadrature method. Figs. 7 and 8 show the relative error of $W_{n}^{m}$ when the number of evaluation points varies. The relative error drastically decreases as the evaluation point increases in the number. $4 \times 4$ evaluation points result in good accuracy, less than $10^{-10}$. It is possible to calculate the accurate spherical harmonic coefficients with a small number of evaluation points.

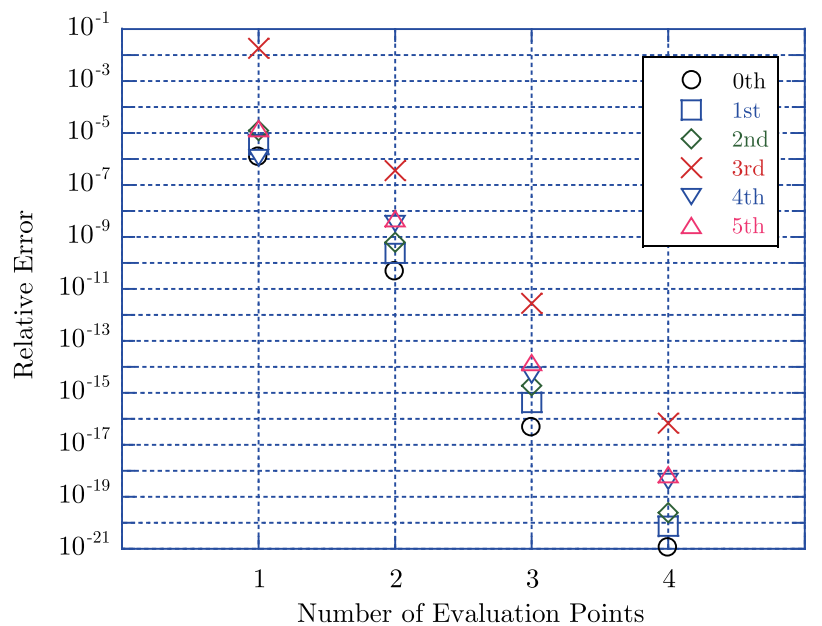

FIG. 10. Accuracy of spherical harmonic coefficients depending on number of evaluation points. 
TABLE IV. Spherical harmonic coefficients of ring coil with large cross section.

\begin{tabular}{cccc}
\hline \hline Order $n$ & 0 & 1 & 2 \\
$C_{z}^{\mathrm{c}}(n, 0)$ & $2.091 \times 10^{-6}$ & $2.476 \times 10^{-5}$ & $1.335 \times 10^{-4}$ \\
Order $n$ & 3 & 4 & 5 \\
$C_{z}^{\mathrm{c}}(n, 0)$ & $5.955 \times 10^{-5}$ & $-5.417 \times 10^{-3}$ & $-4.991 \times 10^{-2}$ \\
\hline \hline
\end{tabular}

\section{B. Ring coil}

The accuracy of the spherical harmonic coefficients of ring coils having small cross-section, like a one-turn coil, and a large one, i.e., a solenoid, is investigated. The calculation of these coefficients of Eqs. (60)-(62) also needs a numerical integration in Eq. (63).

\section{Small cross section}

We can obtain the spherical harmonic coefficients $C^{\mathrm{c}}$ $(n, m)$ and $D^{\mathrm{c}}(n, m)$ for the circular line (one-turn) coil without cross section, using Eqs. (54)-(56). However, it is sometimes necessary to examine the influence of the cross section

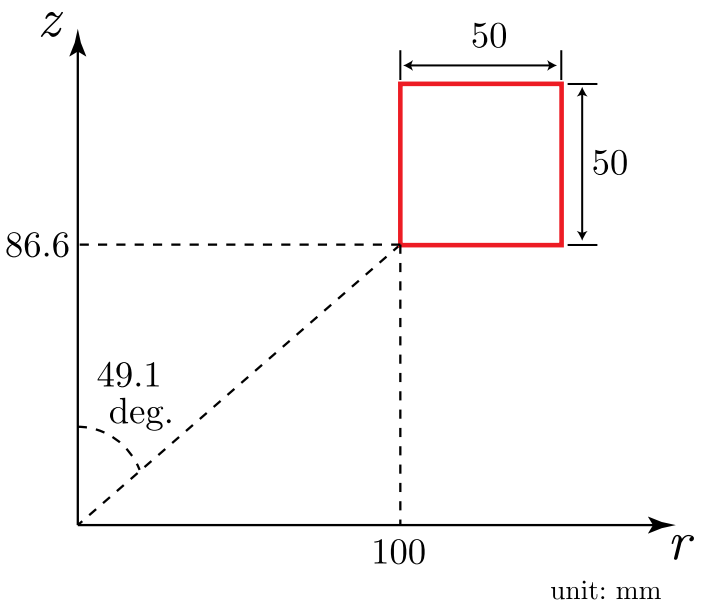

FIG. 11. A circular coil with large cross section as a simple test model.

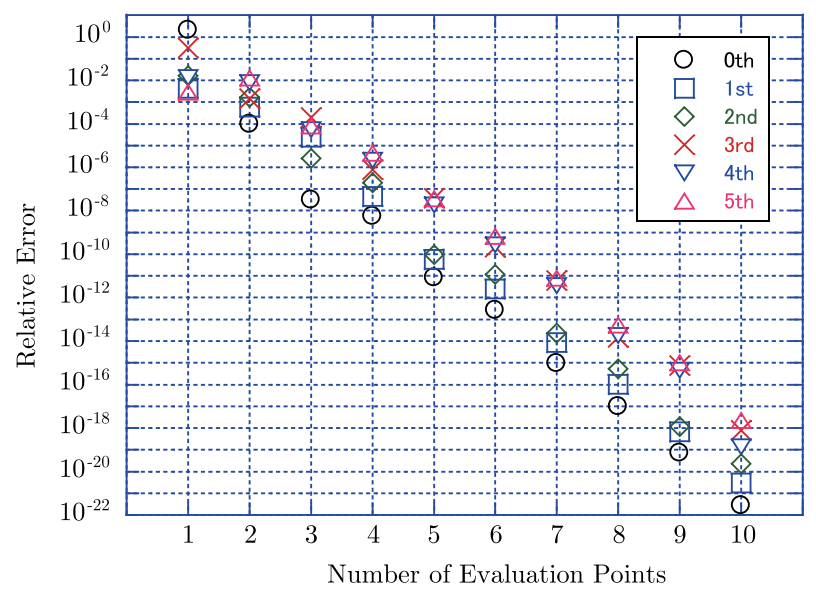

FIG. 12. Accuracy of spherical harmonic coefficients depending on number of evaluation points. The solution with 50 evaluation points is a standard of comparison.
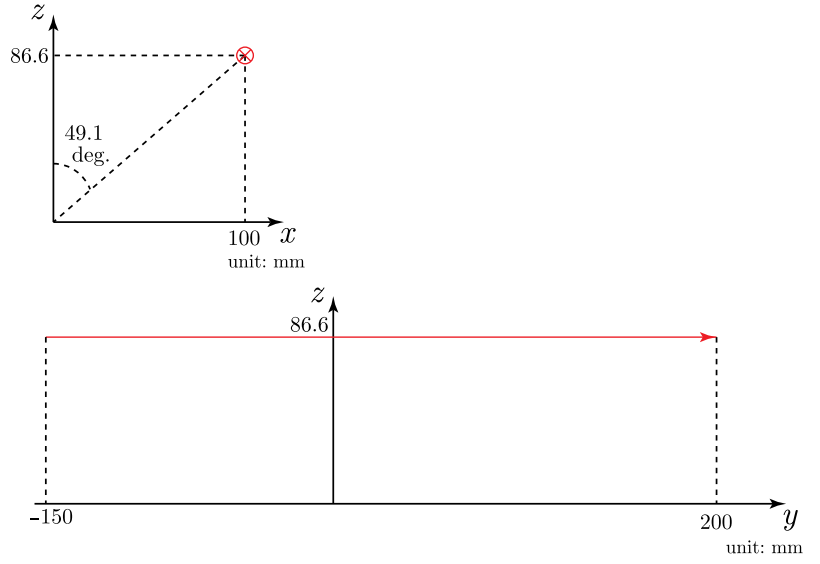

FIG. 13. Straight current test model. $x=100.0 \mathrm{~mm}, z=86.6 \mathrm{~mm}$, $y_{1}=150.0 \mathrm{~mm}, y_{2}=200.0 \mathrm{~mm}, I_{\mathrm{s}}=1 \mathrm{~A}$.

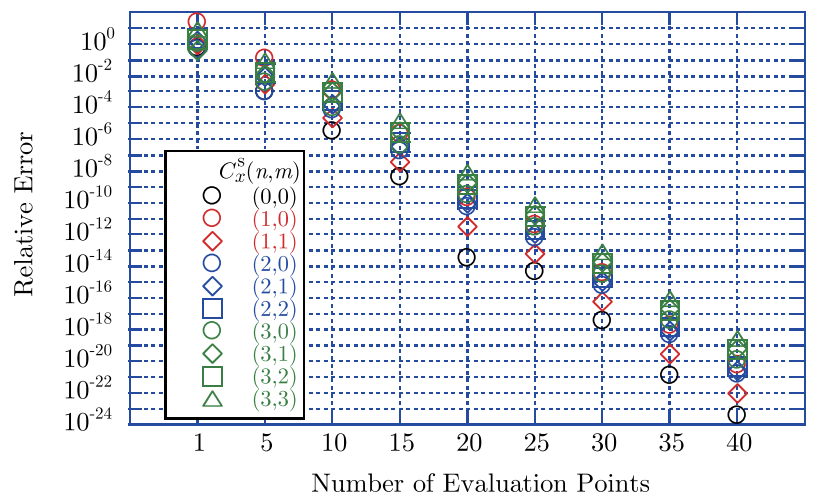

FIG. 14. Relative error of spherical harmonics coefficients $C_{x}^{\mathrm{s}}(n, m)$ of magnetic field generated by line current versus number of evaluation points. The solution with 200 evaluation points is a standard of comparison.

of a wire on the coefficients. Hence, the simple circular coil shown in Fig. 9 is employed for investigation, and the current density is $10^{6} \mathrm{~A} / \mathrm{m}^{2}$ uniformly in the cross section. The number of evaluation points of the Gauss quadrature method varies from 1 to 4 for one dimension, that is, since two-dimensional numerical integration is required, the evaluation points from $1 \times 1$ to $4 \times 4$ are examined. The relative accuracy of $C_{z}^{\mathrm{c}}(n, 0)$ in Eq. (62) on the 0 th to 5 th orders is compared with the coefficients of 50 evaluation points, and

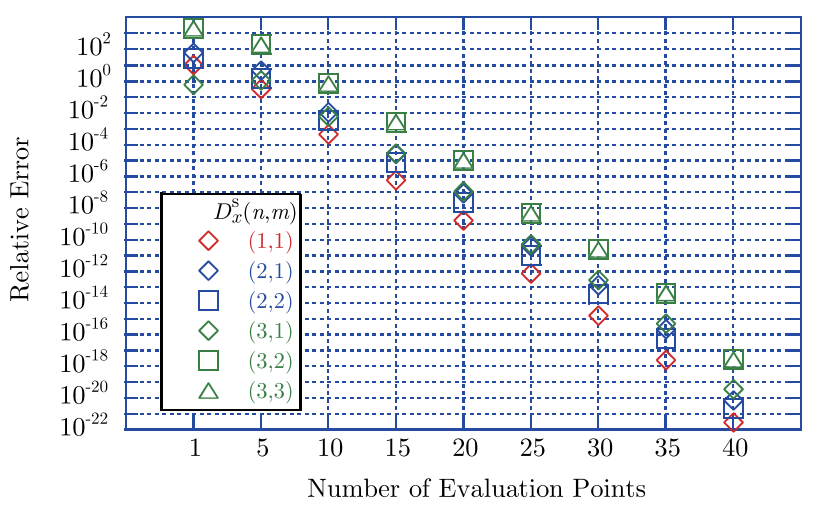

FIG. 15. Relative error of spherical harmonics coefficients $D_{x}^{\mathrm{s}}(n, m)$ of magnetic field generated by line current versus number of evaluation points. The solution with 200 evaluation points is a standard of comparison. 


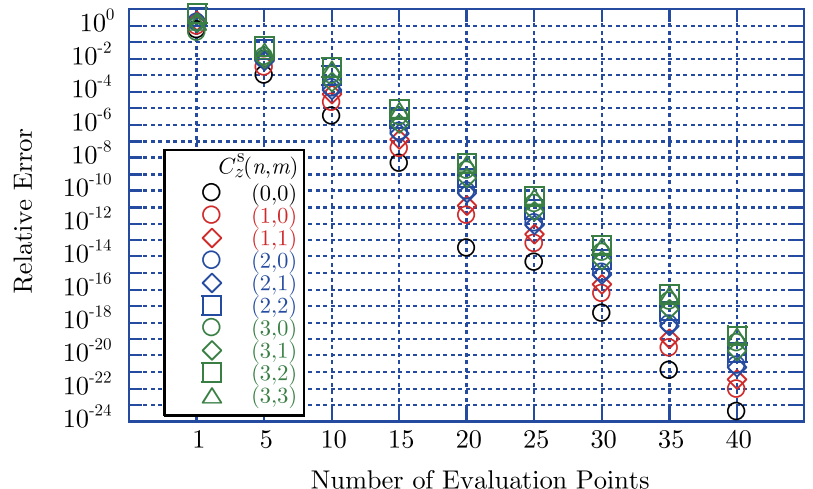

FIG. 16. Relative error of spherical harmonics coefficients $C_{z}^{\mathrm{s}}(n, m)$ of magnetic field generated by line current versus number of evaluation points. The solution with 200 evaluation points is a standard of comparison.

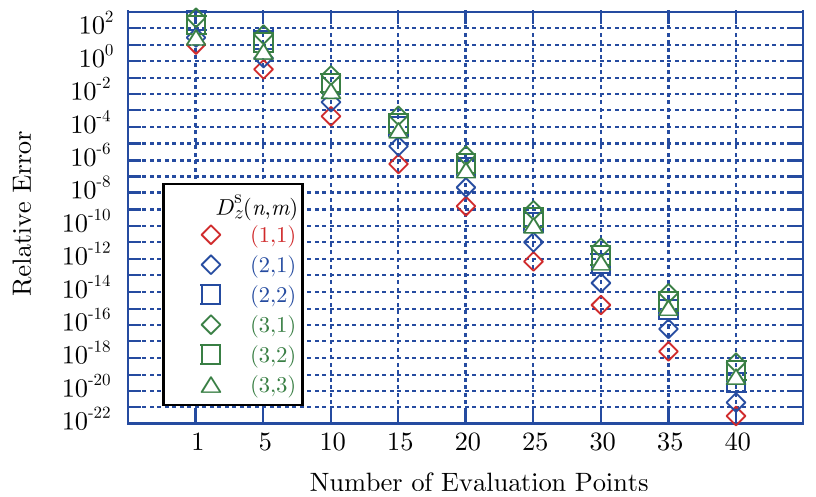

FIG. 17. Relative error of spherical harmonics coefficients $D_{z}^{\mathrm{s}}(n, m)$ of magnetic field generated by line current versus number of evaluation points. The solution with 200 evaluation points is a standard of comparison.

Table III shows the coefficient values. Here, one evaluation point is identical to the circular line coil. Fig. 10 shows the relative error to the number of the evaluation points. The 3 rd-order coefficients are less accurate; however, the accuracy of all the orders exponentially decreases with increase in the number of evaluation points. The Gauss quadrature with three evaluation points is accurate enough for all the orders, less than $10^{-10}$.

TABLE V. Spherical harmonic coefficients $C_{x}^{\mathrm{s}}$ and $D_{x}^{\mathrm{s}}$ of straight line current ( $x$-component).

\begin{tabular}{|c|c|c|c|c|c|}
\hline & \multirow[b]{2}{*}{ C } & \multicolumn{4}{|c|}{$m$} \\
\hline & & 0 & 1 & 2 & 3 \\
\hline \multirow[t]{6}{*}{$n$} & 0 & $-7.84 \times 10^{-7}$ & $\ldots$ & $\ldots$ & $\ldots$ \\
\hline & 1 & $-2.68 \times 10^{-7}$ & $-1.06 \times 10^{-5}$ & $\ldots$ & $\ldots$ \\
\hline & 2 & $1.92 \times 10^{-4}$ & $-6.45 \times 10^{-5}$ & $-1.38 \times 10^{-5}$ & $\ldots$ \\
\hline & 3 & $2.51 \times 10^{-3}$ & $2.48 \times 10^{-4}$ & $-1.23 \times 10^{-4}$ & $-5.08 \times 10^{-6}$ \\
\hline & & \multicolumn{4}{|c|}{$m$} \\
\hline & $D$ & 0 & 1 & 2 & 3 \\
\hline \multirow[t]{4}{*}{$n$} & 0 & 0.00 & $\ldots$ & $\ldots$ & $\ldots$ \\
\hline & 1 & 0.00 & $-4.54 \times 10^{-7}$ & $\ldots$ & $\ldots$ \\
\hline & 2 & 0.00 & $7.02 \times 10^{-7}$ & $-8.07 \times 10^{-7}$ & $\ldots$ \\
\hline & 3 & 0.00 & $2.26 \times 10^{-5}$ & $-4.88 \times 10^{-7}$ & $-5.08 \times 10^{-6}$ \\
\hline
\end{tabular}

TABLE VI. Spherical harmonic coefficients $C_{z}^{\mathrm{s}}$ and $D_{z}^{\mathrm{s}}$ of straight line current ( $z$-component).

\begin{tabular}{|c|c|c|c|c|c|}
\hline & \multirow[b]{2}{*}{ C } & \multicolumn{4}{|c|}{$m$} \\
\hline & & 0 & 1 & 2 & 3 \\
\hline \multirow[t]{6}{*}{$n$} & 0 & $9.05 \times 10^{-7}$ & & $\ldots$ & $\ldots$ \\
\hline & 1 & $1.06 \times 10^{-5}$ & $3.19 \times 10^{-6}$ & $\ldots$ & $\ldots$ \\
\hline & 2 & $4.84 \times 10^{-5}$ & $4.90 \times 10^{-5}$ & $-4.46 \times 10^{-6}$ & $\ldots$ \\
\hline & 3 & $-1.65 \times 10^{-4}$ & $3.11 \times 10^{-4}$ & $2.35 \times 10^{-5}$ & $-1.01 \times 10^{-5}$ \\
\hline & & \multicolumn{4}{|c|}{$m$} \\
\hline & $D$ & 0 & 1 & 2 & 3 \\
\hline \multirow[t]{4}{*}{$n$} & 0 & 0.00 & $\ldots$ & $\ldots$ & $\ldots$ \\
\hline & 1 & 0.00 & $5.25 \times 10^{-7}$ & $\ldots$ & $\ldots$ \\
\hline & 2 & 0.00 & $1.61 \times 10^{-6}$ & $5.72 \times 10^{-8}$ & $\ldots$ \\
\hline & 3 & 0.00 & $6.10 \times 10^{-7}$ & $8.19 \times 10^{-7}$ & $-9.07 \times 10^{-7}$ \\
\hline
\end{tabular}

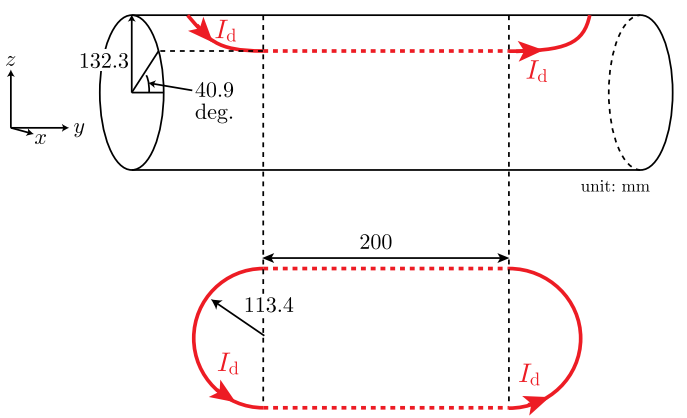

FIG. 18. Schematic view of dipole coil. The current $I_{\mathrm{d}}$ is 1 A. The spherical harmonics coefficients of the curve sections (solid line) are calculated in order to investigate the accuracy.

\section{Large cross section}

The next investigation model is the rectangular coil with larger cross section than the former model. Table IV shows the spherical harmonic coefficients $C_{z}^{\mathrm{c}}(n, 0)$ in Eq. (62). Fig. 11 shows the examination model with the uniform current density of $400 \mathrm{~A} / \mathrm{m}^{2}$. The net current of $1 \mathrm{~A}$ is the same as the former model. The number of the evaluation points varies from 1 to 10 . Fig. 12 shows the relative error depending on the number of the evaluation points. The accuracy deteriorates in the higher orders. With the coil having large cross section, at least 7 evaluation points is necessary to achieve the accuracy less than $10^{-10}$.

\section{Straight line current}

To investigate the accuracy of the spherical harmonic coefficients of a straight current, the relative error of the

TABLE VII. Spherical harmonic coefficients $C_{x}^{\mathrm{d}}(n, m)$ of curve section (x-component).

\begin{tabular}{|c|c|c|c|c|c|}
\hline & & \multicolumn{4}{|c|}{$m$} \\
\hline & & 0 & 1 & 2 & 3 \\
\hline \multirow[t]{4}{*}{$n$} & 0 & 0.00 & $\ldots$ & $\ldots$ & $\ldots$ \\
\hline & 1 & 0.00 & $-2.32 \times 10^{-6}$ & $\ldots$ & $\ldots$ \\
\hline & 2 & 0.00 & $8.80 \times 10^{-6}$ & 0.00 & $\ldots$ \\
\hline & 3 & 0.00 & $4.06 \times 10^{-4}$ & 0.00 & $3.67 \times 10^{-3}$ \\
\hline
\end{tabular}


TABLE VIII. Spherical harmonic coefficients $D_{y}^{\mathrm{d}}(n, m)$ of curve section (y-component).

\begin{tabular}{|c|c|c|c|c|c|}
\hline & & \multicolumn{4}{|c|}{$m$} \\
\hline & & 0 & 1 & 2 & 3 \\
\hline \multirow[t]{4}{*}{$n$} & 0 & 0.00 & $\ldots$ & $\ldots$ & $\ldots$ \\
\hline & 1 & 0.00 & $-5.48 \times 10^{-6}$ & $\ldots$ & $\ldots$ \\
\hline & 2 & 0.00 & $-2.64 \times 10^{-5}$ & 0.00 & $\ldots$ \\
\hline & 3 & 0.00 & $1.86 \times 10^{-4}$ & 0.00 & $1.91 \times 10^{-3}$ \\
\hline
\end{tabular}

TABLE IX. Spherical harmonic coefficients $C_{z}^{\mathrm{d}}(n, m)$ of curve section $(z$ component).

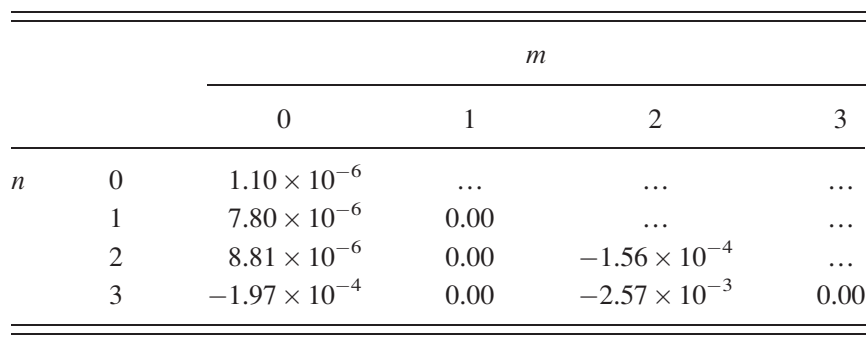

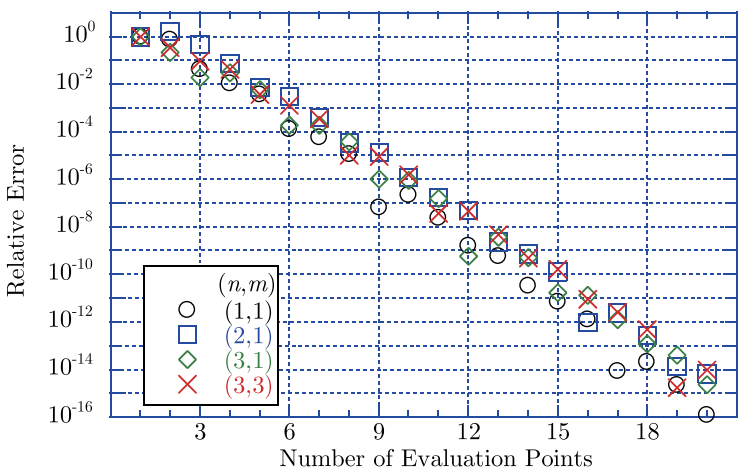

FIG. 19. Relative error of spherical harmonics coefficients $C_{x}^{\mathrm{d}}(n, m)$ of magnetic field generated of curve section by increment of the number of evaluation points. The solution with 200 evaluation points is a standard of comparison.

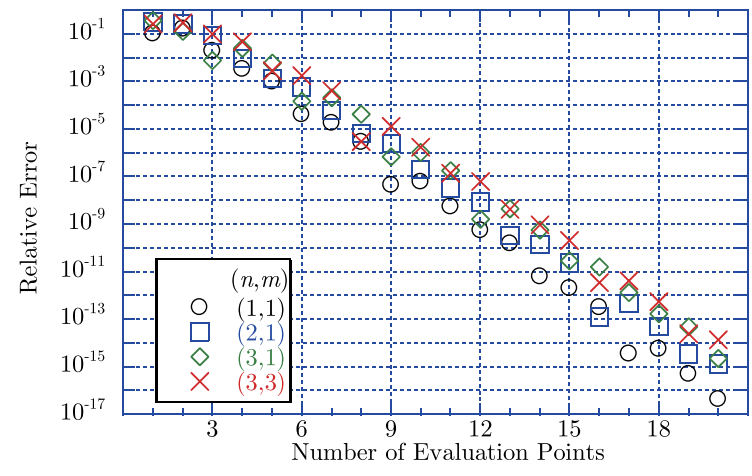

FIG. 20. Relative error of spherical harmonics coefficients $D_{y}^{\mathrm{d}}(n, m)$ of magnetic field generated of curve section by increment of the number of evaluation points. The solution with 200 evaluation points is a standard of comparison.

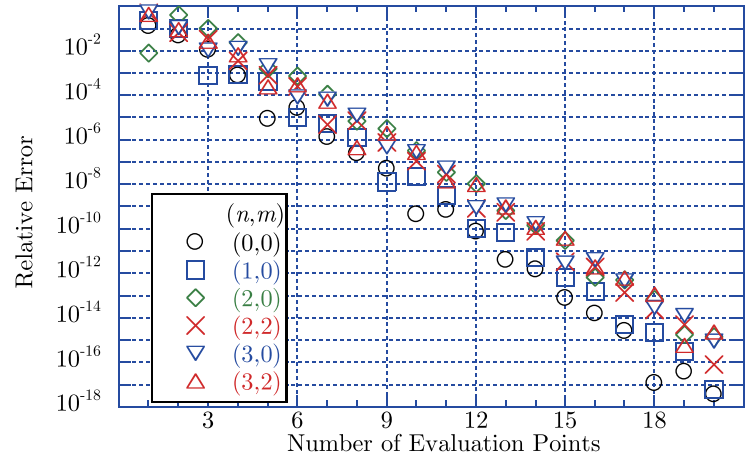

FIG. 21. Relative error of spherical harmonics coefficients $C_{x}^{\mathrm{d}}(n, m)$ of $z$-component magnetic field generated of curve section by increment of the number of evaluation points. The solution with 200 evaluation points is a standard of comparison.

coefficients is calculated depending on the number of the evaluation points of the Gauss quadrature method. Fig. 13 shows a straight current model, and the current is 1 A. Figs. 14 and 15 represent the relative error of the coefficients $C_{x}^{\mathrm{s}}(n, m)$ and $D_{x}^{\mathrm{s}}(n, m)$ in Eq. (72), respectively, when the evaluation points increases from 1 to 50. Figs. 16 and 17 also show the relative error of the coefficients $C_{z}^{\mathrm{s}}(n, m)$ and $D_{z}^{\mathrm{s}}(n, m)$ in Eq. (73), respectively. In these figures, the calculation with 200 evaluation points is regarded as a standard. Tables V and VI show the $x$ - and $z$-components of the spherical harmonic coefficients. Entirely the accuracy is not sufficient, so at least 30 evaluation points is required. The accuracy on the straight current is much worse than that of the ring coils.

\section{Dipole magnet}

A numerical integration is needed to calculate the spherical harmonic coefficients $C^{\mathrm{d}}(n, m)$ and $D^{\mathrm{d}}(n, m)$ of the magnetic field generated by a dipole magnet. For investigation of the accuracy of the spherical harmonic coefficients, those of curve sections are calculated, because the previous section describes on these of the straight line section.

Fig. 18 shows the schematic view of the curve section of the dipole magnet, and the current of 1 A flows. The coefficients $D^{\mathrm{d}}(n, m)$ of $x$ - and $z$-components and $C^{\mathrm{d}}(n, m)$ of $y$-component of the magnetic field are 0 due to the symmetry of the configuration. Tables VII-IX show the coefficients of $x$-, $y$-, and $z$-components, respectively. The relative errors are shown in Figs. 19-21, where 200 evaluation points are assumed as the standard, when the evaluation point in the Gauss quadrature method increases from 1 to 20 . All the coefficients exponentially decrease, so 16 evaluation points are necessary for the relative error below $10^{-10}$.

\section{CONCLUSION}

When a magnet is shimmed for a highly homogeneous field application, it is important to evaluation the field homogeneity with the coefficients of spherical harmonic series of the generated field. However, the active and passive shimmings to date comensate the only $z$-component coefficients of spherical harmonic series. Hence, the formulas of the coefficients were derived in the $z$-component field for various kinds 
of coils. In this paper, I derive the spherical harmonic coefficients of the entire components of magnetic field generated by a magnetized ferromagnetic material piece, a circular line coil, a circular coil with volume, a straight line current, and a dipole coil. Since all the formulas include an integration form, the relations between the relative error of the spherical harmonic coefficients and the number of evaluation points in the Gauss quadrature method are shown. Although the numerical integration in the straight line current indicates a low convergence, a good accuracy is achieved with a small number of evaluation points in the other cases.

When a highly homogeneous magnet is designed or an active or passive shimming is performed, it is effective to employ the derived formulas in an optimization algorithm. ${ }^{13,16-18,21,22}$ The spherical harmonic coefficients presented in this paper facilitate active shimming for various kinds of coils by eliminating the higher terms of the entirecomponent coefficients than the 0th one. On the other hand, in the case of passive shimming, it is difficult to eliminate the higher terms of the coefficients. The magnetic moment of a magnetized ferromagnetic material piece has to be computed because of its nonlinearity and saturation. Therefore, the magnetic moment method ${ }^{23,24}$ is effective in computing the magnetic moment. The passive shimming is done by combining the magnetic moment method with an optimization method. ${ }^{22}$

Recently, a few magnets are designed to generate a magnetic field tilted from the $z$-axis. The forms of the spherical harmonic coefficients presented in this paper and the previous ones ${ }^{4-17}$ cannot be easily used for shimming design computation. Hence, the rotational transform of the spherical harmonic coefficients is a usable way, and the method of the rotational transform is presented in the paper. ${ }^{25}$ The tilted magnetic field can be also homogenized with the rotational transform technique.

I have been developing a shimming technique including the presented coefficients, the optimization method, the magnetic moment, and the rotational transform technique. The result of passive shimming in the previous paper ${ }^{21}$ was obtained using the formulas (38)-(48) presented in this paper, the magnetic moment method, ${ }^{23,24}$ the rotation transform, ${ }^{25}$ and the genetic algorithm. ${ }^{26,27}$ The developed technique will be applied to more complicated magnet, such as an open type MRI and a racetrack magnet of accelerator, for active and passive shimming. In addition, the formulas of the spherical harmonic coefficient for the iron piece and the various coils will give a large contribute to the field of magnet design.

\section{ACKNOWLEDGMENTS}

The author thanks Professor Yukikazu Iwasa and Dr. Seungyong Hahn of the Francis Bitter Magnet Laboratory, Massachusetts Institute of Technology.

\section{APPENDIX A: LEGENDRE FUNCTIONS}

Tables X and XI give the list of the Legendre functions and the associated Legendre functions, respectively.
TABLE X. Legendre functions.

$$
\begin{aligned}
& P_{0}(\sigma)=1 \\
& P_{1}(\sigma)=\sigma \\
& P_{2}(\sigma)=1 / 2\left(3 \sigma^{2}-1\right) \\
& P_{3}(\sigma)=1 / 2\left(5 \sigma^{3}-3 \sigma\right) \\
& P_{4}(\sigma)=1 / 8\left(35 \sigma^{4}-30 \sigma^{2}+3\right) \\
& P_{5}(\sigma)=1 / 8\left(63 \sigma^{5}-70 \sigma^{3}+15 \sigma\right) \\
& P_{6}(\sigma)=1 / 16\left(231 \sigma^{6}-315 \sigma^{4}+105 \sigma^{2}-5\right) \\
& P_{7}(\sigma)=1 / 16\left(429 \sigma^{7}-693 \sigma^{5}+315 \sigma^{3}-35 \sigma\right) \\
& P_{8}(\sigma)=1 / 128\left(6435 \sigma^{8}-12012 \sigma^{6}+6930 \sigma^{4}-1260 \sigma^{2}+35\right) \\
& \text { where } \sigma=\cos \theta
\end{aligned}
$$

TABLE XI. Associated Legendre functions.

$$
\begin{aligned}
& P_{1}^{1}(\sigma)=\sin \theta \\
& P_{2}^{1}(\sigma)=3 \sin \theta \cos \theta \\
& P_{2}^{2}(\sigma)=3 \sin ^{2} \theta \\
& P_{3}^{1}(\sigma)=3 / 2 \sin \theta\left(5 \cos ^{2} \theta-1\right) \\
& P_{3}^{2}(\sigma)=15 \sin ^{2} \theta \cos \theta \\
& P_{3}^{3}(\sigma)=15 \sin ^{3} \theta \\
& P_{4}^{1}(\sigma)=5 / 2 \sin \theta\left(7 \cos ^{3} \theta-3 \cos \theta\right) \\
& P_{4}^{2}(\sigma)=15 / 2 \sin ^{2} \theta\left(7 \cos ^{2} \theta-1\right) \\
& P_{4}^{3}(\sigma)=105 \sin ^{3} \theta \cos \theta \\
& P_{4}^{4}(\sigma)=105 \sin ^{4} \theta \\
& P_{5}^{1}(\sigma)=15 / 8 \sin \theta\left(21 \cos ^{4} \theta-14 \cos ^{2} \theta+1\right) \\
& P_{5}^{2}(\sigma)=105 / 2 \sin ^{2} \theta\left(3 \cos ^{3} \theta-\cos \theta\right) \\
& P_{5}^{3}(\sigma)=105 / 2 \sin ^{3} \theta\left(9 \cos ^{2} \theta-1\right) \\
& P_{5}^{4}(\sigma)=945 \sin ^{4} \theta \cos \theta \\
& P_{5}^{5}(\sigma)=945 \sin ^{5} \theta \\
& P_{6}^{1}(\sigma)=21 / 8 \sin \theta\left(33 \cos ^{5} \theta-30 \cos ^{3} \theta+5 \cos \theta\right) \\
& P_{6}^{2}(\sigma)=105 / 8 \sin ^{2} \theta\left(33 \cos ^{4} \theta-18 \cos ^{2} \theta+1\right) \\
& P_{6}^{3}(\sigma)=315 / 2 \sin ^{3} \theta\left(11 \cos ^{3} \theta-3 \cos \theta\right) \\
& P_{6}^{4}(\sigma)=345 / 2 \sin ^{4} \theta\left(11 \cos ^{2} \theta-1\right) \\
& P_{6}^{5}(\sigma)=10395 \sin ^{5} \theta \cos \theta \\
& P_{6}^{6}(\sigma)=10395 \sin ^{6} \theta \\
& P_{7}^{1}(\sigma)=7 / 16 \sin \theta\left(429 \cos ^{6} \theta-495 \cos ^{4} \theta+135 \cos ^{2} \theta-5\right) \\
& P_{7}^{2}(\sigma)=63 / 8 \sin ^{2} \theta\left(143 \cos ^{5} \theta-110 \cos ^{3} \theta+15 \cos \theta\right) \\
& P_{7}^{3}(\sigma)=315 / 8 \sin ^{3} \theta\left(143 \cos ^{4} \theta-66 \cos ^{2} \theta+3\right) \\
& P_{7}^{4}(\sigma)=3465 / 2 \sin ^{4} \theta\left(13 \cos ^{3} \theta-3 \cos \theta\right) \\
& P_{7}^{5}(\sigma)=10395 / 2 \sin ^{5} \theta\left(13 \cos ^{2} \theta-1\right) \\
& P_{7}^{6}(\sigma)=135135 \sin ^{6} \theta \cos \theta \\
& P_{7}^{7}(\sigma)=135135 \sin ^{7} \theta \\
& P_{8}^{1}(\sigma)=9 / 16 \sin \theta\left(715 \cos ^{7} \theta-1001 \cos ^{5} \theta+385 \cos ^{3} \theta\right. \\
& -35 \cos \theta) \\
& P_{8}^{2}(\sigma)=315 / 16 \sin ^{2} \theta\left(143 \cos ^{6} \theta-143 \cos ^{4} \theta+33 \cos ^{2} \theta-1\right) \\
& P_{8}^{3}(\sigma)=3465 / 8 \sin ^{3} \theta\left(39 \cos ^{5} \theta-26 \cos ^{3} \theta+3 \cos \theta\right) \\
& P_{8}^{4}(\sigma)=10395 / 8 \sin ^{4} \theta\left(65 \cos ^{4} \theta-26 \cos ^{2} \theta+1\right) \\
& P_{8}^{5}(\sigma)=135135 / 2 \sin ^{5} \theta\left(5 \cos ^{3} \theta-\cos \theta\right) \\
& P_{8}^{6}(\sigma)=135135 / 2 \sin ^{6} \theta\left(15 \cos ^{2} \theta-1\right) \\
& P_{8}^{7}(\sigma)=2027025 \sin ^{7} \theta \cos \theta \\
& P_{8}^{8}(\sigma)=2027025 \sin ^{8} \theta \\
& \text { where } \sigma=\cos \theta
\end{aligned}
$$


APPENDIX B: PARTIAL DIFFERENTIAL OF FUNCTION $\boldsymbol{T}_{n m}^{\prime}$

The partial differential of $T_{n m}^{\prime}$ in the spherical coordinate is $\frac{\partial T_{n m}^{\prime}}{\partial x}=\sin \alpha \cos \psi \frac{\partial T_{n m}^{\prime}}{\partial r_{0}}+\frac{\cos \alpha \cos \psi}{r_{0}} \frac{\partial T_{n m}^{\prime}}{\partial \alpha}-\frac{\sin \psi}{r_{0} \sin \alpha} \frac{\partial T_{n m}^{\prime}}{\partial \psi}$,

$$
\begin{aligned}
& \frac{\partial T_{n m}^{\prime}}{\partial y}= \sin \alpha \sin \psi \frac{\partial T_{n m}^{\prime}}{\partial r_{0}}+\frac{\cos \alpha \sin \psi}{r_{0}} \frac{\partial T_{n m}^{\prime}}{\partial \alpha} \\
&+\frac{\cos \psi}{r_{0} \sin \alpha} \frac{\partial T_{n m}^{\prime}}{\partial \psi}, \\
& \frac{\partial T_{n m}^{\prime}}{\partial z}=\cos \alpha \frac{\partial T_{n m}^{\prime}}{\partial r_{0}}-\frac{\sin \alpha}{r_{0}} \frac{\partial T_{n m}^{\prime}}{\partial \alpha} .
\end{aligned}
$$

When $r>r_{0}$,

$$
\begin{aligned}
\frac{\partial T_{n m}^{\prime}}{\partial r_{0}} & =n r_{0}^{n-1} P_{n}^{m}(\cos \alpha) \cos [m(\phi-\psi)], \\
\frac{\partial T_{n m}^{\prime}}{\partial \alpha}= & r_{0}^{n}\left[m \cot \alpha P_{n}^{m}(\cos \alpha)-P_{n}^{m+1}(\cos \alpha)\right] \\
& \times \cos [m(\phi-\psi)], \\
\frac{\partial T_{n m}^{\prime}}{\partial \psi} & =m r_{0}^{n} P_{n}^{m}(\cos \alpha) \sin [m(\phi-\psi)] .
\end{aligned}
$$

The following equations are derived by the substitution of Eqs. (B4)-(B6) to (B1)-(B3).

$$
\begin{aligned}
\frac{\partial T_{n m}^{\prime}}{\partial x}= & \frac{r_{0}^{n-1}}{2}\left\{\cos [(m+1) \psi-m \phi]\left[(n-m) \sin \alpha \times P_{n}^{m}(\cos \alpha)-\cos \alpha P_{n}^{m+1}(\cos \alpha)\right]\right. \\
& \left.+\cos [(m-1) \psi-m \phi]\left[(n-m) \sin \alpha \times P_{n}^{m}(\cos \alpha)-\cos \alpha P_{n}^{m+1}(\cos \alpha)+\frac{2 m}{\sin \alpha} P_{n}^{m}(\cos \alpha)\right]\right\} \\
\frac{\partial T_{n m}^{\prime}}{\partial y}= & \frac{r_{0}^{n-1}}{2}\left\{\sin [(m+1) \psi-m \phi]\left[(n-m) \sin \alpha \times P_{n}^{m}(\cos \alpha)-\cos \alpha P_{n}^{m+1}(\cos \alpha)\right]\right. \\
& \left.-\sin [(m-1) \psi-m \phi]\left[(n-m) \sin \alpha \times P_{n}^{m}(\cos \alpha)-\cos \alpha P_{n}^{m+1}(\cos \alpha)+\frac{2 m}{\sin \alpha} P_{n}^{m}(\cos \alpha)\right]\right\} \\
& \frac{\partial T_{n m}^{\prime}}{\partial z}=r_{0}^{n-1} \cos [m(\phi-\psi)]\left\{(n-m) \cos \alpha P_{n}^{m}(\cos \alpha)+\sin \alpha P_{n}^{m+1}(\cos \alpha)\right\} .
\end{aligned}
$$

We have the following relations from: ${ }^{6}$

$$
\begin{gathered}
(n-m) \sin \alpha P_{n}^{m}(\cos \alpha)-\cos \alpha P_{n}^{m+1}(\cos \alpha)=-P_{n-1}^{m+1}(\cos \alpha) \\
-P_{n-1}^{m+1}(\cos \alpha)+\frac{2 m}{\sin \alpha} P_{n}^{m}(\cos \alpha)=(n+m)(n+m-1) P_{n-1}^{m-1}(\cos \alpha) \\
(n-m) \cos \alpha P_{n}^{m}(\cos \alpha)+\sin \alpha P_{n}^{m+1}(\cos \alpha)=(n+m) P_{n-1}^{m}(\cos \alpha) .
\end{gathered}
$$

We can derive Eqs. (8)-(10) from (B7)-(B9) using (B10)-(B12).

Similarly, when $r<r_{0}$,

$$
\begin{gathered}
\frac{\partial T_{n m}^{\prime}}{\partial r_{0}}=-\frac{1}{r_{0}^{n+2}}(n+1) P_{n}^{m}(\cos \alpha) \times \cos [m(\phi-\psi)], \\
\frac{\partial T_{n m}^{\prime}}{\partial \alpha}=\frac{1}{r_{0}^{n+1}}\left[m \cot \alpha P_{n}^{m}(\cos \alpha)-P_{n}^{m+1}(\cos \alpha)\right] \times \cos [m(\phi-\psi)], \\
\frac{\partial T_{n m}^{\prime}}{\partial \psi}=m \frac{1}{r_{0}^{n+1}} P_{n}^{m}(\cos \alpha) \sin [m(\phi-\psi)] .
\end{gathered}
$$

The following equations are also derived from Eqs. (B13)-(B15) substituting into Eqs. (B1)-(B3).

$$
\begin{aligned}
\frac{\partial T_{n m}^{\prime}}{\partial x}= & -\frac{1}{2 r_{0}^{n+2}}\left\{\cos [(m+1) \psi-m \phi]\left[(n+m+1) \times \sin \alpha P_{n}^{m}(\cos \alpha)+\cos \alpha P_{n}^{m+1}(\cos \alpha)\right]\right. \\
& \left.+\cos [(m-1) \psi-m \phi]\left[(n+m+1) \sin \alpha \times P_{n}^{m}(\cos \alpha)+\cos \alpha P_{n}^{m+1}(\cos \alpha)-\frac{2 m}{\sin \alpha} P_{n}^{m}(\cos \alpha)\right]\right\}
\end{aligned}
$$




$$
\begin{aligned}
\frac{\partial T_{n m}^{\prime}}{\partial y}= & -\frac{1}{2 r_{0}^{n+2}}\left\{\sin [(m+1) \psi-m \phi]\left[(n+m+1) \times \sin \alpha P_{n}^{m}(\cos \alpha)+\cos \alpha P_{n}^{m+1}(\cos \alpha)\right]\right. \\
& \left.-\sin [(m-1) \psi-m \phi]\left[(n+m+1) \sin \alpha \times P_{n}^{m}(\cos \alpha)+\cos \alpha P_{n}^{m+1}(\cos \alpha)-\frac{2 m}{\sin \alpha} P_{n}^{m}(\cos \alpha)\right]\right\}
\end{aligned}
$$

$$
\begin{aligned}
\frac{\partial T_{n m}^{\prime}}{\partial z}= & -\frac{1}{r_{0}^{n+2}} \cos [m(\phi-\psi)]\{(n+m+1) \cos \alpha \\
& \left.\times P_{n}^{m}(\cos \alpha)-\sin \alpha P_{n}^{m+1}(\cos \alpha)\right\} .
\end{aligned}
$$

We can derive Eqs. (11)-(13) from (B16)-(B18) using (B19)-(B21).

$$
(n+m+1) \sin \alpha P_{n}^{m}(\cos \alpha)+\cos \alpha P_{n}^{m+1}(\cos \alpha)=P_{n+1}^{m+1}(\cos \alpha)
$$

$$
\begin{aligned}
& P_{n+1}^{m+1}(\cos \alpha)-\frac{2 m}{\sin \alpha} P_{n}^{m}(\cos \alpha) \\
& \quad=-(n-m+1)(n-m+2) P_{n+1}^{m-1}(\cos \alpha)
\end{aligned}
$$

TABLE XII. List of $G E_{n}(\gamma, \eta)$.

$$
\begin{aligned}
& G E_{2}(\gamma, \eta)=\frac{4 \pi}{10} \frac{1}{2 a_{1}^{2} \eta}\left(K_{1}^{3 / 2}-K_{3}^{3 / 2}\right) \\
& G E_{4}(\gamma, \eta)=\frac{4 \pi}{10} \frac{1}{24 a_{1}^{4} \eta^{3}}\left[K_{1}^{3 / 2}\left(2+3 K_{2}+15 K_{2}^{2}\right)-K_{3}^{3 / 2}\left(2+3 K_{4}+15 K_{4}^{2}\right)\right] \\
& G E_{6}(\gamma, \eta)=\frac{4 \pi}{10} \frac{1}{240 a_{1}^{6} \eta^{5}}\left[K_{1}^{3 / 2}\left(8+12 K_{2}+15 K_{2}^{2}-70 K_{2}^{3}+315 K_{2}^{4}\right)\right. \\
& \left.-K_{3}^{3 / 2}\left(8+12 K_{4}+15 K_{4}^{2}-70 K_{4}^{3}+315 K_{4}^{4}\right)\right] \\
& G E_{8}(\gamma, \eta)=\frac{4 \pi}{10} \frac{1}{896 a_{1}^{8} \eta^{7}}\left[K _ { 1 } ^ { 3 / 2 } \left(16+24 K_{2}+30 K_{2}^{2}+35 K_{2}^{3}+315 K_{2}^{4}\right.\right. \\
& \left.-2079 K_{2}^{5}+3003 K_{2}^{6}\right) \\
& \left.-K_{3}^{3 / 2}\left(16+24 K_{4}+30 K_{4}^{2}+35 K_{4}^{3}+315 K_{4}^{4}-2079 K_{4}^{5}+3003 K_{4}^{6}\right)\right] \\
& G E_{10}(\gamma, \eta)=\frac{4 \pi}{10} \frac{1}{11520 a_{1}^{10} \eta^{9}}\left[K _ { 1 } ^ { 3 / 2 } \left(128+192 K_{2}+240 K_{2}^{2}+280 K_{2}^{3}\right.\right. \\
& \left.+315 K_{2}^{4}-2772 K_{2}^{5}+42042 K_{2}^{6}-128700 K_{2}^{7}+109395 K_{2}^{8}\right) \\
& -K_{3}^{3 / 2}\left(128+192 K_{4}+240 K_{4}^{2}+280 K_{4}^{3}+315 K_{4}^{4}-2772 K_{4}^{5}\right. \\
& \left.\left.+42042 K_{4}^{6}-128700 K_{4}^{7}+109395 K_{4}^{8}\right)\right] \\
& G E_{12}(\gamma, \eta)=\frac{4 \pi}{10} \frac{1}{33792 a_{1}^{12} \eta^{11}}\left[K _ { 1 } ^ { 3 / 2 } \left(256+384 K_{2}+480 K_{2}^{2}+560 K_{2}^{3}\right.\right. \\
& +630 K_{2}^{4}+693 K_{2}^{5}+9009 K_{2}^{6}-167310 K_{2}^{7}+875160 K_{2}^{8} \\
& \left.-1616615 K_{2}^{9}+969969 K_{2}^{10}\right)-K_{3}^{3 / 2}\left(256+384 K_{4}+480 K_{4}^{2}\right. \\
& +560 K_{4}^{3}+630 K_{4}^{4} 693 K_{4}^{5}+9009 K_{4}^{6}-167310 K_{4}^{7} \\
& \left.\left.+875169 K_{4}^{8}-1616615 K_{4}^{9}+969969 K_{4}^{10}\right)\right] \\
& \text { where } K_{1}=\frac{1}{1+\eta^{2}}, K_{2}=\frac{\eta^{2}}{1+\eta^{2}}, K_{3}=\frac{\gamma^{2}}{\gamma^{2}+\eta^{2}}, K_{4}=\frac{\eta^{2}}{\gamma^{2}+\eta^{2}}
\end{aligned}
$$

$$
\begin{aligned}
& (n+m+1) \cos \alpha P_{n}^{m}(\cos \alpha)-\sin \alpha P_{n}^{m+1}(\cos \alpha) \\
& \quad=(n-m+1) P_{n}^{m+1}(\cos \alpha) .
\end{aligned}
$$

\section{APPENDIX C: LIST OF FUNCTION $G_{E_{n}}(\gamma, \boldsymbol{\eta})$}

Table XII gives the list of $G E_{n}(\gamma, \eta)\left(=G(\gamma, \eta) E_{n}\right){ }^{20}$

${ }^{1}$ Y. Iwasa, Case Studies in Superconducting Magnets: Design and Operational Issues, 2nd ed. (Springer, 2009).

${ }^{2}$ Y. Lvovsky and P. Jarvis, IEEE Trans. Appl. Supercond. 15, 1317 (2005).

${ }^{3}$ M. Augath, P. Heiler, S. Kirsch, and L. R. Schad, J. Magn. Reson. 200, 134 (2009).

${ }^{4}$ F. Roméo and D. I. Hoult, Magn. Reson. Med. 1, 44 (1984).

${ }^{5}$ J. E. C. Williams, IEEE Trans. Nucl. Sci. 31, 994 (1984).

${ }^{6}$ W. M. Schmidt, R. R. Huson, W. W. Mackay, and R. M. Rocha, IEEE Trans. Magn. 27, 1681 (1991).

${ }^{7}$ L. K. Forbes, S. Crozier, and D. M. Doddrell, IEEE Trans. Magn. 33, 4405 (1997).

${ }^{8}$ C. J. Snape-Jenkinson, S. Crozier, and L. K. Forbes, ANZIAM J. 43, 375 (2002).

${ }^{9}$ D. I. Hoult and D. Lee, Rev. Sci. Instrum. 56, 131 (1985).

${ }^{10}$ A. Belov, V. Bushuev, M. Emelianov, V. Eregin, Y. Severgin, S. Sytchevski, and V. Vasiliev, IEEE Trans. Appl. Supercond. 5, 679 (1995).

${ }^{11} \mathrm{H}$. Zhao and S. Crozier, Meas. Sci. Technol. 13, 198 (2002).

${ }^{12}$ H. S. Lopez, F. Liu, E. Weber, and S. Crozier, IEEE Trans. Magn. 44, 394 (2008).

${ }^{13} \mathrm{~S}$. Noguchi and A. Ishiyama, in Proceedings of 15th International Conference on Magnet Technology (1998), Vol. 2, p. 1414.

${ }^{14}$ F. Liu, J. Zhu, L. Xia, and S. Crozier, IEEE Trans. Appl. Supercond. 21, 60 (2011).

${ }^{15}$ H. Zhao and S. Crozier, Meas. Sci. Technol. 13, 2047 (2002).

${ }^{16}$ S. Noguchi and A. Ishiyama, IEEE Trans. Magn. 33, 1904 (1997).

${ }^{17}$ S. Noguchi and A. Ishiyama, IEEE Trans. Appl. Supercond. 7, 439 (1997).

${ }^{18}$ S. Kakugawa, N. Hino, A. Komura, M. Kitamura, H. Takeshima, T. Yatsuo, and H. Tazaki, IEEE Trans. Appl. Supercond. 14, 1624 (2004).

${ }^{19}$ J. Voccio, S. Hahn, D. K. Park, J. Ling, Y. Kim, J. Bascuñán, and Y. Iwasa, IEEE Trans. Appl. Supercond. 23, 4300804 (2013).

${ }^{20}$ D. B. Montgomery, Solenoid Magnet Design: The Magnetic and Mechanical Aspects of Resistive and Superconducting Systems (John Wiley \& Sons Ltd., 1969).

${ }^{21}$ S. Noguchi, S. Kim, S. Hahn, and Y. Iwasa, IEEE Trans. Magn. 50, 7014904 (2014).

${ }^{22}$ S. Noguchi, S. Hahn, and Y. Iwasa, IEEE Trans. Appl. Supercond. 24, 4400904 (2014).

${ }^{23}$ Y. Takahashi, S. Wakao, and A. Kameari, J. Appl. Phys. 99, 08 H904 (2006).

${ }^{24}$ Y. Takahashi, C. Matsumoto, and S. Wakao, IEEE Trans. Magn. 43, 1277 (2007).

${ }^{25}$ M. A. Blanco, M. Flórez, and M. Bermejo, J. Mol. Struct. 419, 19 (1997).

${ }^{26}$ D. E. Goldberg, Genetic Algorithms in Serach, Optimization, and Machine Learning (Addison-Wesley, 1989).

${ }^{27}$ S. Noguchi and A. Ishiyama, IEEE Trans. Magn. 32, 2655 (1996). 